\title{
A Behavioral Sequence Analyzing Framework for Grouping Students in an E-learning System ${ }^{\text {光 }}$
}

\author{
Tao Xie, Qinghua Zheng, Weizhan Zhang \\ MOEKLINNS Lab, Department of Computer Science and Technology, Xi'an Jiaotong University, Xi'an, 710049 China
}

\begin{abstract}
Grouping of students benefits the formation of virtual learning communities, and contributes to collaborative learning space and recommendation. However, the existed grouping criteria are mainly limited in the learning portfolios, profiles, and social attributes etc. In this paper, we aim to build a unified framework for grouping students based on the behavioral sequences and further predicting which group a newcomer will be. The sequences are represented as a series of behavioral trajectories. We discuss a shape descriptor to approximately express the geometrical information of trajectories, and then capture the structural, micro, and hybrid similarities. A weighted undirected graph, using the sequence as a node, the relation as an edge, and the similarity as the weight, is constructed, on which we perform an extended spectral clustering algorithm to find fair groups. In the phase of prediction, an indexing and retrieval scheme is proposed to assign a newcomer to the corresponding group. We conduct some preliminary experiments on a real dataset to test the availability of the framework and to determine the parameterized conditions for an optimal grouping. Additionally, we also experiment on the grouping prediction with a synthetic data generator. Our proposed method outperforms the counterparts and makes grouping more meaningful.
\end{abstract}

Keywords: Grouping students, Behavioral sequence, Behavioral trajectory, Similarity measure 2015 MSC: 71.35.-y, 71.35.Lk, 71.36.+c

\section{Introduction}

All the time, grouping of students is a popular topic not only in classroom but also in many e-learning environments. In the classical education, the educators often deliberately design some team projects to promote students' collaborative and inquiry skills [1] or to influence their affections and values [2]. To this end, grouping is treated as a crucial process since its quality has much effect on the pedagogical objectives. As the computer and internet are widely used in education, the significance of grouping doesn't decline with the increase of transactional distance [3] between the teachers and students. Instead, its status has become more prominent due to the larger demand of personalization and the higher targets of collaboration and communication. Generally, two reasons lead to grouping. On the one hand, grouping in e-learning benefits the formation of so-called virtual learning communities [4] where the students

\footnotetext{
This research was partially supported by the National Science Foundation of China under Grant Nos. 61472317, 61428206, 61221063, 61472315, 91218301, the MOE Innovation Research Team No. IRT13035, the Coordinator Innovation Project for the Key Lab of Shaanxi Province under Grant No.2013SZS05-Z01, the Online Education Research Foundation of MOE Research Center for Online Education under Grant Nos. 2016YB165, 2016YB169, the Natural Science Basic Research Plan in Shaanxi Province of China Nos. 2016JM6027, 2016JM6080, and the Project of China Knowledge Centre for Engineering Science and Technology.

Email addresses: graber@mail.xjtu.edu.cn (Tao Xie), qhzheng@mail.xjtu.edu.cn (Qinghua Zheng), zhangwzh@mail.xjtu.edu.cn (Weizhan Zhang)
}

within a community share the common interest, competence, or goal. On the other hand, grouping acts as a compromise of pursuing effect and avoiding cost in the personalized procedure. Although one student one program pattern may be more effective in learning, it's usually too expensive to hand out one program to each student especially when the user community is very large. In this case, grouping is a smart choice considering that students in the same group may have similar characteristics, interests, preferences, or abilities, while the ones in different groups may not have much in common. Besides, grouping makes special sense to collaborative learning space [5], along with recommendations [6] for future research.

Although the topic of grouping has been discussed for several years, most of them are based on the learning portfolios, profiles, social attributes, and the rising behavior-driven approaches. Little work has focused on the behavioral sequences composed of students' operation series as well as the insightful information behind. The modern learning theory indicates that students are the central part of the cognitive activity and the active constructors of the learning environment. Their actively constructed processes, i.e. interactions, may vary with the individuals' states, experiences, affections, expectations, competence, and so on. A learning behavior is generated as the interaction occurs, which is often stamped by time and accompanied with an operation type. The behaviors in durations would constitute all kinds of behavioral sequences depending on different users. We are curious that whether we would have an opportunity to extract underlying information that characterizes learning 
and even process grouping for a more homogenous structure. As the vigorous development of information technology, such behavioral sequences are easy to be captured but insufficient to be reported.

Our assumption is similar to Hilliard et al. (1992) that two groups of students would develop habits and preferences that cause them to perform in somewhat different ways owing to the diversities of behavioral styles [7]. The behavioral style is defined as a habitual behavior pattern, where we could group students by a certain differential thresholds. Previous studies used to adopt the sequential mining technology to address this discriminating task [8], which attempted to find the frequen$t$ patterns based on their occurrences. Nevertheless, it is insufficient to follow the sequences with interest only because a student's behavior is motivated by the complex inner process, where we may not get pedagogically-sound results until we take the deeper meaning behind the data into consideration. Moreover, the frequency is not a critical factor comparing with the students' interests in some cases. For example, an event with high frequency but low interest is invalid, and an event with low frequency but high interest, on the contrary, often counts. This intuition conforms to the paradoxical experimental conclusion in literature [9] that the more frequent a pattern is the more trivial or valueless it tends to be. Therefore, the sequential mining methods couldn't help us to acquire the desired results largely due to different data structure and different professional implications.

This study presents a view to analyze the behavioral sequences for the goal of grouping, which does not exist in other's work as far as we know. We attempt to identify students of which behavioral styles should be clustered in the same group and further estimate which group a newcomer will be as he/she styles his/her sequences. The first issue is a problem of similarity measurement. Although it has well been studied in a lot of fields including e-learning, nearly no one has considered the characteristics of sequences and data interaction simultaneously. The second issue is in essence a problem of prediction depending on the similarity models. To address these two issues effectively, we primarily take four actions. Firstly, we convert the sequences to behavioral trajectories. Secondly, we convert the calculation problem of behavior similarity to the one of trajectory similarity. Thirdly, we convert the problem of clustering to that of graph partitioning. And lastly, we convert the problem of complex prediction to that of simple character matching and distance measurement. The energy of the matrix is more concentrated after using Principle Component Analysis (PCA). The most promising group of the query sequence is the one that has the smallest distance to it.

The main contributions of this paper are:

(i) We propose a framework for grouping students based on their behavioral sequences logged in system. The grouping has dual meanings. One is dividing the sequences into several groups, and the other is assigning a new one into the most matching group. The central part of the framework is exploring the similarities of sequences and designing the prediction algorithm in terms of the newly defined data models.

(ii) An entirely different approach is presented to compute the similarity of sequences. We for the first time propose the conception of micro similarity of learning behaviors as well as the corresponding calculating method.

(iii) We propose a novel on-line predicting mechanism, i.e. indexing and retrieval, to effectively assign a student to the most matching group on a basis of the newly logged data and the historical models. This mechanism dedicates to condensing space of the models and carefully selecting important points, thus makes the space out of sparseness and keeps most of the features intact.

(iv) We conduct a series of extensive experiments on the learning behavioral logs collected during a whole school term to verify the availability of the proposed framework. We compare the gaps among approaches, study how to choose parameters for a better grouping, and examine the ability of online prediction compared to two common baselines via a synthetic behavior generator.

The rest of the paper is structured as follows. We discuss related works in Section 2. Section 3 introduces the research problems. Our proposed method is detailed in section 4. In section 5, we demonstrate the preliminary experimental results. Section 6 draws a conclusion and outlooks the future research.

\section{Related Work}

Grouping has extensive applications, one of which is recommender system. Users are first partitioned into groups of similar ones, then recommendations are produced based on the characteristics of group members without searching the whole group base. For example, to recommend learning activities, Wu et al. proposed to match similar users based on a tree matching method and develop a novel similarity measure to handle the category issue of grouping [10]; to recommend point-ofinterest locations, Chen et al. made groups according to users' demographics and frequently visited positions [11]. Without been exclusively emphasized, one sees that grouping has been treated as an important component of recommender system. In this section, we briefly review the previous studies mainly from the e-learning fields with three categories: grouping strategies, clustering approaches, and similarity measures.

Grouping techniques have been extensively researched recently. The big significance of grouping in teaching and learning environment is beyond all question in that there are considerable literatures have studied the positive effect of grouping on learning [12][13]. In traditional classroom, grouping is more based on the teachers' knowledge about students or class performance [14]. In computer-supported learning, the criteria for grouping are unrestricted. Many people, on a basis of the online test or usage logs, focused on the students' leaning portfolios, profiles, and social attributes etc. In literature [15] for example, the authors did a work on the students' portfolios. The portfolios usually comprised rich information figures, where students with similar portfolios were assembled in the same group while different groups revealed diversified characteristics. Their strategy was to collect the students' assignment scores, exam scores, and learning records, and then they used data mining techniques to obtain the grouping results. The work mainly depended on 
the teachers' observation about the students' learning activities, and most data sources originated from the students' mastery of knowledge. Thus their approaches lack strength to offer proper solutions to the problems we are currently focusing on.

The research on grouping based on the students' profiles is appeared in literature [16]. The profiles were modeled as the students' features such as personal details, course details, interests, team roles, preferences, trust ranking, and so on. They tried to achieve optimal grouping by satisfying some task constraints. However, the work is unable to handle the behavior data concerned in this paper. In addition, grouping with social attributes is also a hot topic. The social attributes comprise multi facets including social characteristics, relationships, interaction processes, social goals, and planning. For example, Graf et al. (2006) proposed an approach to form heterogeneous groups based on personality traits and social performance [17]. They concerned more on the macro dimension and left the micro undetected. Therefore, this grouping method is also not we are interested in. Actually, the grouping criteria are much more than those described above. Nevertheless, it is a challenging work to propose an both effective and pedagogicallysound grouping strategy since the optimal grouping is always controlled by many factors involving the students' skills, interests, preferences, competence, and affections etc.

Recently, a behavior-driven method has become an emerging branch, where the researchers concern more exquisite factors. This approach approximates to ours, however the big gap lies in the definition of learning behavior thus leads to quite different solutions. For example, Cocea et al. focused on such behaviors that students used the system to solve problems of mathematical generalization [18]. They grouped students through case-based reasoning and clustering according to the process of completing tasks. The most related work appears in literature [19]. The authors proposed to use data mining methods and visualization tools to elicit interesting learning behavior patterns, which they analyzed them from two perspectives action frequency and action sequences with a goal of unobtrusively and ubiquitously learning from a students previous experiences and characteristics. However, they mainly considered the physical characteristics of sequences but failed to concentrate on the data interaction. Moreover, they didn't handle the paradox of frequency and importance. Therefore, the meaningful grouping may not be obtained.

Other important descriptions of behaviors are click-stream [20] and web session [21] that reflect users' behavior patterns. However, it is still a blank towards the students' operation series. In this study, the behavior is defined as a student's habitual operations when he/she interacts with the system. We extend the current research on grouping by taking into account of the meaning, temporal characteristic, and data interaction. The research theme provides an alternative grouping scheme, which seldom relies on test or requires less about students' information. Besides, we incorporate our grouping technique into a complete framework so as to not only can we group historical data but also we are able to predict the newcomers. Such work does not exist in other literatures for all we know.

The core of grouping is clustering, while the base of clus- tering is similarity measurement. Firstly, the clustering is used to recognize the natural groups within a class of entities in an unsupervised way. Previous studies on clustering accumulated a superb collection of standard algorithms. K-means, for example, is a representative clustering method, and is widely used for its conciseness and efficiency. However, such methods are usually limited due to the specific research conditions and strategies. In this paper, grouping is required to be more fair and meaningful, thus many methods may be eclipsed. We follow the graph clustering [22]. The graph is a structure formed by a set of vertices and edges. Each object corresponds to a vertex and the interaction between objects corresponds to an edge. The task of clustering is to group the vertices into clusters with heterogeneous characteristics. Secondly, the similarity is the quantitative degree of how close two objects are under the mathematical framework. Although there are a great number of similarity measures encountered in different fields, they have respective limitations and the choice depends on one's aims and efficiency. The most popular metric Euclidean distance, for example, is commonly used to evaluate the proximity of objects in the low dimensional space. It fits for numeric attributes, but limits that the attributes of objects having the same size. In the present paper, we intend to measure the similarities of behavioral sequences. The physical sequence is a basic factor, it's nevertheless not enough to find ground truth if we don't consider more insightful information behind the data. The similarity degree is much influenced by the key points in sequences, where detecting these points is playing a critical role and not involved in others work. As an example, suppose a behavior A happens shortly after it happened, does it mean A is an important behavior or it should be determined by the local ingredient in the interaction region? The answer seems obvious since the former will affect the latter if they are in an appropriate interaction region and an action will decay naturally as time went by unless it's reinforced by its previous neighbors. Therefore, the routine methods are helpless to handle this affair of similarity computation.

In summary, grouping in terms of the behavioral sequences has not been studied sufficiently. Such sequential data is usually treated as a series of numerical signals, ignoring the hidden information behind. When the data is endowed with a specific professional implication, we take a second thought about how to group meaningfully. The existed work did not consider the multi-faceted models of data, as well as not present the grouping technique based on the behavioral sequences in a complete framework.

\section{Problem Statement}

Generally speaking, a full expression of an event can be simply stated as somebody does something at sometime. Therefore we assume that a well-designed system is capable of logging at least four basic components: 1) the subject of behavior; 2) the outcome of behavior; 3) the time when the behavior is triggered; and 4) the materials the subject focuses on. A subject is a student who studies using a unique virtual identity number. The time component records when a specified operation occurs. The 
outcome, i.e. behavioral sequence, represents a student's usage process associated with time. And the materials represent the learning content a student is interested in.

Thus, four basic sets are formed, i.e. the set of users, the set of behavioral sequences, the set of timing, and the set of materials. There exist multiple complex mappings between the subjects and objects. For example, a user can learn multiple materials in a duration, and a material can also be learnt by multiple users. In the interaction of users and materials, the behaviors of different styles are generated. This paper mainly concerns the behavioral data and leaves the research with respect to materials in the future. The behaviors, because of their specific background and semantic information, represent learning process which can be reconstructed approximately from the behavioral logs. Then we are able to find their commonness and individuality and further use them for educational purposes.

Some essential definitions are given:

Definition 1.(Learning Log) A learning log contains information about the person, time, and behavioral outputting sequence. One can learn when the person had what kind of operation at a nearer time. The learning log of a person is $L_{p}=<\left(d_{1}, s_{1}\right),\left(d_{2}, s_{2}\right), \ldots,\left(d_{n}, s_{n}\right)>$, where $d_{i}, 1 \leqslant i \leqslant n$, denotes the time when the sequence occurs, say the date, and $s_{i}, 1 \leqslant i \leqslant n$, the daily interaction sequence.

Definition 2.(Learning Behavior) A learning behavior $l b$ is produced by a student when he/she interacts with the system. Let $\left\{o_{1}, o_{2}, \ldots, o_{n}\right\}$ be a set of operation types, $T=\left\{t_{1}, t_{2}, \ldots, t_{n}\right\}$ be a set of primitive time units. A $l b=(o, t), o \in O, t \in T$, records an operation $o$ occurs at time stamp $t$. The point $(o, t)$ in the two-dimensional space is also called a behavioral sample point in loco.

Definition 3.(Operation Type) A $l b$ corresponds to a specified operation type, explaining the meaning of the behavior occurrence. Let $O$ denotes the set of all possible operation types. An operation $o \in O$ that happens during a period of time $\left[t_{s}, t_{e}\right]$ is denoted as $(o, \Delta t)$, where $t_{s}$ is the start time and $t_{e}$ the end time, $\Delta t=t_{e}-t_{s}$. We call $\Delta t$ the duration of operation $o$ and $o$ the operation with time interval $\Delta t$.

Definition 4.(Behavioral sequence) A behavioral sequence $b s$ comprises a series of tuples $<\left(o_{1}, t_{1}\right),\left(o_{2}, t_{2}\right), \ldots,\left(o_{n}, t_{n}\right)>$. The portion of a sequence is called its subsequence. Given $A=<a_{1}, a_{2}, \ldots, a_{n}>$ and $B=<b_{1}, b_{2}, \ldots, b_{m}>, A$ is the subsequence of $B$, if and only if $|A| \leqslant|B|, A \subseteq B$ and there exist integers $1 \leqslant i_{1}<\ldots<i_{n} \leqslant m$ such that $b_{k}=a_{i_{k}}$ for all $k$, where $1 \leqslant k \leqslant n$. In other words, the elements in $A$ also exist in $B$ while keeping their order and consecutiveness, and an injective mapping $\pi:\{i, i+1, \ldots, j\} \rightarrow\{1,2, \ldots, m\}, 1 \leqslant i \leqslant j \leqslant m$ is strictly satisfied. Conversely, $B$ is the mother sequence of $A$.

Definition 5.(Behavioral Style) The behavioral style refers to the pattern of observable behavioral sequences. It has key characteristics that represent the habitual actions and behavior dispositions emerged at the time when a student interacts with the system, whose function is to explain the gap in behavioral sequences.

\section{Method}

Students' behavioral sequences imply habits and preferences. Those who have similar behavioral sequences would share the similar behavioral styles and should be grouped together. This consideration results in the following three issues to be solved: 1) how to express learning behavioral sequences of different students; 2) how to cluster the sequences into several groups according to their characteristics; and 3) how to adaptively assign a new query sequence to a suitable group as he/she styles his/her sequences. In this section, we will outline our research framework in 4.1 , followed by a detailed similarity measure in 4.2 , then we extend a clustering algorithm in 4.3 and make a prediction in 4.4 .

\subsection{Research Framework}

When each behavioral sample point is treated as a specific symbol in a set, the sequences composed of the symbols and the interaction relations are endowed with special information under the guidance of pedagogy. This is much like the protein or gene sequence in bioengineering that when the real meaning is considered, it is not only a set of chemical bonds but controls the functions of organisms. When we detect one of the similar sequences has a specific function, the other sequences probably have the similar function too. Providing that the behaviors are driven by the internal learning motivations, we are able to discover the unique behavioral styles from the students' sequences. The similarity measure and clustering method are the center of grouping, while the prediction is an important aspect of grouping, which is properly assigning a new query user into the corresponding group. We address it by calculating the distance between each query user and the models. However, it is not easy to solve all the problems at one stroke since one should consider the meaning of behaviors, grouping performance, and the prediction ability etc. The research framework is presented in Fig. 1. The framework is phased to offline analysis and online prediction, divided by the horizontal long dotted line. The main steps of offline phase are:

(i) Assembling the scatters $H D$ in continuous time slices to be $B T$ that has a certain shape.

(ii) Computing the similarity between trajectories and constructing a $S M$ and a graph.

(iii) Partitioning the graph with a spectral clustering algorith$\mathrm{m}$ with a threshold to make it group.

(iv) Reducing dimensionality and extracting features on each cluster with PCA. The result matrices $M_{1} \sim M_{n}$ are called moulds.

As for the new coming query data in the online phase, the processing is basically in line with the early stage of offline phase. The difference is that the query data is added a salient points selection SPS, which is a method to keep the most important data intact. Subsequently, the query sequence is adapted to the moulds and assigned to the one with the smallest distance. 


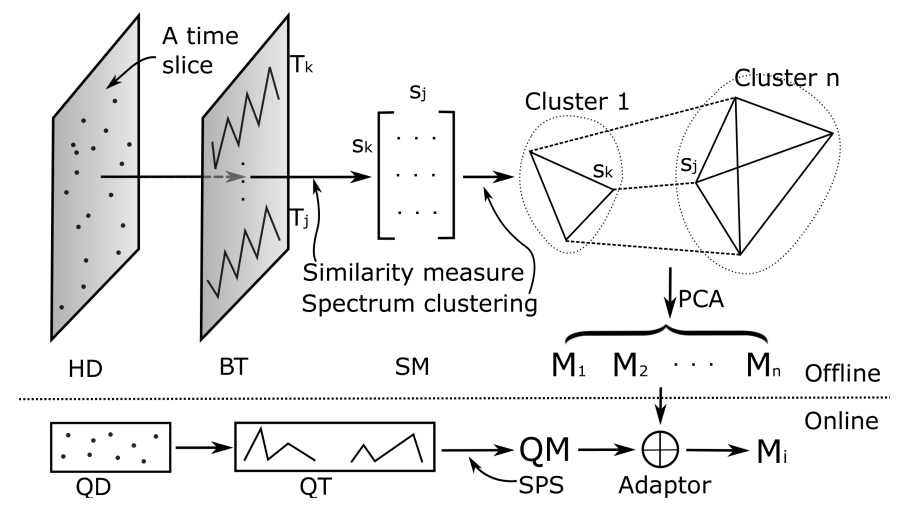

Fig. 1. The proposed research framework. $H D$ : History Data. $B T$ : Behavioral Trajectory. SM: Similarity Matrix. $Q D$ : Query Data. $Q T$ : Query Trajectory. QM: Query Matrix. SPS: Salient Points Selection.

\subsection{Similarity Exploration}

The first task we have to tackle is to cluster different behavioral sequences according to the similarities. A sequence can be viewed as a string, where each element in the string represents an operation type. The main challenge is how to measure the similarity of sequences traced by students. In this section, we first describe the data models so that one can better understand the sequence features, then we introduce a shape estimating method, namely shape descriptor, to visually present the data. Thirdly, we compute the similarities from structural, micro and hybrid aspects.

\subsubsection{Data Models}

Building models of sequences can help us better understand the data features and implications. We discuss them from three angles.

\section{(A) Physical Model.}

A physical sequence is the natural sequence which is made up of random behavioral events chronologically disregarding the context meaning of each element and maintaining interindependence between elements. It is the foundation of the work and constitutes the essential shapes of behavioral trajectories. A behavioral sequence includes at least two physical sequences. One represents the stream of operation types, the other comprises a series of time intervals between operations. We assume that the events' occurrences are driven by the students' real demands. Then they would share a certain regularity, and the implicit demands lead to a behavioral randomness as well. It is noting that the regularity does not conflict with the randomness, since the randomness naturally survives on the regularity while the regularity is the special case in the randomness.

Regularity 1. Short Loops, interpreted as a student's frequent and periodical actions. Let $S=<s_{1}, s_{2}, \ldots, s_{n}>$ be a physical sequence, and let $S^{\prime}=<s_{i}, s_{i+1}, \ldots, s_{j}>$ be the subsequence of $S$, where $1 \leqslant i<j \leqslant n$. A short loop occurs if the following two conditions hold:

(i) $s_{i}=s_{j}$ and $\forall s_{k} \neq s_{i}, i<k<j$.

(ii) There exists another subsequence $S^{\prime \prime}=<s_{p}, s_{p+1}, \ldots, s_{q}>$ , $1 \leqslant p<q \leqslant n$, such that $s_{i}=s_{p}, s_{i+1}=s_{p+1}, \ldots, s_{j}=s_{q}$.
The (i) accounts for the start and the end positions of the subsequence should be the same so that a closed circle is ensured while the remaining positions should be completely different to guarantee it is the smallest circle. The (ii) accounts for the non-uniqueness of the circle, representing the loop property of the circle in the whole sequence. For example $A B A$ is a circle, while $A B A B$ is a short loop with two occurrences and we denote it as $(A B)_{2}$ for simplicity.

Regularity 2. Recurrence. When an operation occurs, we expect it would recur very soon. This feature can be interpreted as probability distribution of random behaviors. It can be estimated by its past occurrences. Given a training model and a sequence $b_{1} b_{2} \ldots b_{i-1}$, the likelihood of observation $b_{i}$ can be denoted as $P\left(b_{i} \mid b_{1} b_{2} \ldots b_{i-1}\right)$. This idea of recurrence over time has a very substantial theoretical and practical basis. From the perspective of behavioral scientists for example, the behaviors of a subject encompass a number of repeated sequences of acts which is the essential substrate for the existence of a habit [23]. Marta C. Gonzlez et al.(2008) claimed in their paper published in Nature that humans follow simple reproducible patterns despite of the diversity of their backgrounds.

Regularity 3. Position Skipping, which means two operations of the same type are less likely to occur successively. For example when a student watches a course video, there is usually a pause action between two adjacent play actions, and an end mark representing the terminal of current action will be inevitably inserted between two adjacent drag actions. Another example is material browsing. After ending up current browsing and before switching to the next one, some intermediate actions are commonly existed such as going back to the previous level or clicking some additional tips.

\section{(B) Temporal Model.}

Each student's behavioral style has a certain temporal characteristics depending on their habits and interests. The term habit indicates that something happens repeatedly over the course of time, while interest literally means the order and frequency of an event's occurrence. The time is an important indicator, where the temporal data is constituted as considering the associations between events in the time dimension. Usually the temporal data is represented on a basis of discrete time points, where a pair of time points defines a time interval starting at an earlier point and ending at a latter point. The temporal is computed by comparing the time relationships of intervals. In the context of this research, some interferential elements (or so-called background noises) exist unavoidably in the behavioral sequences due to randomness. The amount of time spent on different elements can be used for measuring their respective impacts to determine whether to be identified or filtered. Therefore we expect the temporal information to be discriminatory to sequences. For each pair of actions $a_{i}$ and $a_{j}$, the temporal relation $\operatorname{TR}\left(a_{i}, a_{j}\right)$ can be calculated by quantifying their time contributions. However, the consideration of temporal factor survives on the people's definitions in a specific application. We hold the view that there is a distance restriction because $T R$ between two distant actions is 0 , which means we will focus on temporal relations in a finite range. 


\section{(C) Link Model.}

Skinner (1938) acknowledged that human behavior is mainly the operational behavior composed of operational reflection. Operational behavior acts on the environment and generates the outcomes, so it is a mean of reinforcing stimulus. The changes of behaviors are caused by the stimulating factors. A behavior will be strengthened if its occurrence is confirmed during the meaning construction, or conversely it will be weakened. We claim that there exist a certain links between behaviors especially when they are in neighborhood. Take short loops $(A B)_{2}$ for example, if $A$ happens shortly after $(A B)_{2}$ happened, we conclude that the impact of $A$ will increase as $A$ is reinforced by the environment short loop and recurrence. The theoretic underpinning behind this idea also reflects in the work of psychologist K. Leven about the argument that the behavior is a function of the person in their environment, see [24].

On the basis of the three models described above, in what follows, we provide a three-level research scheme. On the first level (coarse level), we define a shape descriptor, which is perceptual knowledge about the data (4.2.2). The second level (middle level) takes the structural features of sequences into account. And on the third level (fine level), we focus on the data interaction and temporal evolution (4.2.4).

\subsubsection{Shape Descriptor of the Data}

To start with, we discuss an approximated method to visually present the data, so that one can roughly monitor the sources from students. A behavior is always stamped by time. The time is continuous and the behavior is discrete. Each pair of time and behavior can be viewed as a point in the two-dimensional space. Linearly connecting these time-behavior pairs one by one chronologically, an unsmoothed broken line is constituted. The sequences from multiple users could respectively generate their lines in such a way. The term approximated is used because it fails to express the full information. Instead, it concentrates more on the physical model of sequences. However, it's beneficial to the analysis of the other two models.

The online learning behavior varies from person to person. When the time is sufficient and the student performs actively (disregarding the case of exiting the running system shortly after landed and the case of doing nothing despite he/she is in), the behaviors of a student are exclusively represented by such a line. We call the line Behavioral Trajectory $(B T)$ formally described in Definition 6. We mapping the $B T$ into a two-dimensional coordinate system, where the horizontal coordinate represents the time stretching, labeled by categories the behaviors belong to, and the longitudinal coordinate represents the expression degrees of the behaviors, quantified by the time overheads that the behaviors have consumed. The time is an important indicator. Generally speaking, a behavior is more important if its time is longer with an exception that the behavior itself is invalid. The volume depends on specific behavioral types and how people feel it interesting. Thus the values may have a large rangeability. For example, the operations of transitional types tend to have lower values, while the valid learning events may have higher values. If the duration is anomaly large, the behavior is invalid on the contrary. Hence, to reduce the affect of absolute value of elements, we normalize the value to fall between 0 and 1 . We choose the max-minimum normalization. Given a sequence $S=<s_{1}, s_{2}, \ldots, s_{n}>$, its normalized sequence is represented as $S^{\prime}=<\operatorname{norm}\left(s_{1}\right), \operatorname{norm}\left(s_{2}\right), \ldots, \operatorname{norm}\left(s_{n}\right)>$. Here,

$$
\operatorname{norm}\left(s_{i}\right)=\left\{\begin{array}{l}
\frac{s_{i}-\min (s \mid s \in S)}{\max (s \mid s \in S)-\min (s \mid s \in S)}, \\
\text { if } \max (s \mid s \in S)<t h ; \\
\frac{s_{i}-\min (s \mid s \in S)}{\text { th }-\min (s \mid s \in S)}, \\
\text { if } \max (s \mid s \in S)>=\text { th and } s_{i}<t h ; \\
\frac{\text { th }-\min (s \mid s \in S)}{\text { th-min }(s \mid s \in S)}, \text { otherwise. }
\end{array}\right.
$$

where $i \in\{1,2, \ldots, n\}$, th a user defined threshold, exceeding which the value will be cut off.

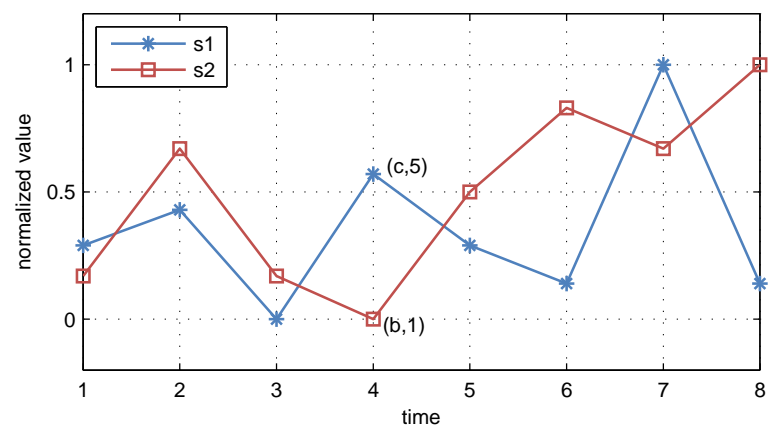

Fig. 2. A simple example of behavior trajectories given $s 1=<(b, 3)$, $(e, 4),(a, 1),(c, 5),(e, 3),(b, 2),(a, 8),(c, 2)>$ and $s 2=<(a, 2),(b, 5),(e, 2)$, $(b, 1),(a, 4),(c, 6),(a, 5),(e, 7)>$.

Definition 6. (Behavioral Trajectory, BT) Let $I=\left\{\left(o_{1}, t_{1}\right)\right.$, $\left.\left(o_{2}, t_{2}\right), \ldots,\left(o_{n}, t_{n}\right)\right\}$ be the element set of a behavioral sequence with continuous time, where $t$ is a time unit and the 2-tuple $\left(o_{i}, t_{i}\right)$ means operation $o_{i}$ occurs at time $t_{i}$. We describe the behavior distribution using the horizontal axis and the time investment using the vertical axis. Then $\left(o_{i}, t_{i}\right)$ represents a point in x-y coordinate system. Connecting $\left(o_{i}, t_{i}\right)$ and $\left(o_{i+1}, t_{i+1}\right)$, we get a segment $s_{i}$. Consequently, $I$ is expressed by an unsmoothed broken line, i.e. behavioral trajectory, in the plane, denoted as $S=\left\{\left(s_{1}, \Delta t_{1}\right),\left(s_{2}, \Delta t_{2}\right), \ldots,\left(s_{n}, \Delta t_{n}\right)\right\}$.

A simple example of behavioral trajectories is given in Fig. 2. The task of analyzing the similarity is translated into comparing the shapes of trajectories. It's natural to connect it with time series data. The time series is a collection of observations made chronologically with some properties including large data size, high dimensionality, and continuous update [25]. In the domain of AI, there are a lot of algorithms for computing similarity. In between, k-means is popular and simple, generally used to cluster data points on the basis of Euclidean distance function (EDF). However, the EDF does not work well in the time series data [26] since it has a strict limitation that sequences are of the same length, which is difficult to meet in most cases. Furthermore, the samples in sequences are not often at the fixed time stamps, but they have a time shift. Under this circumstance, Dynamic Time Warping (DTW) has proven to be efficient. DTW 
is a well-known approach to measure the distance of time series by dynamic programming technique. It builds a distance matrix according to Euclidean distance function and a cumulative distance of a minimum of three surrounding neighbors [27]. It can be represented by

$$
M(i, j)=\left|a_{i}-b_{j}\right|^{2}+\min (M(i-1, j), M(i, j-i), M(i-1, j-1)),
$$

where the first part on the right hand of the equality is $L_{2}-$ norms of two points, and the second part is a minimum cumulative distance of upper, left, and upper left. The main drawback of DTW is the calculation complexity especially when the sequences are too long. The longer the sequences are, the bigger the matrix is, thus the more the complexity will be.

Therefore, the discussed approaches may not fit since the behavioral trajectory in this research has the following characteristics:

(i) The distribution of behaviors over the horizontal direction is not equidistant. A real behavioral trajectory is not as ideal as the case in Fig. 2 that each point is naturally aligned to the same time stamp but they have a time offset.

(ii) The lengths of trajectories are not equal. This means any method that relies on the Euclidean distance measurement is not appropriate. It requires to compare a trajectory $S_{1}$ to another longer trajectory $S_{2}$, and find all the subsequences in $S_{2}$ that match $S_{1}$. The overhead is very high because $S_{1}$ should be placed at every possible positions within $S_{2}$.

(iii) Unlike most time series data, the approximate degree of behavioral sample points relies on the nodes in their neighborhood. On the one hand, considering point $p$ in two trajectories, when it has the same precursor $q$, the segment connecting $p$ and $q$ may be more similar than other cases. On the other hand, when a sample point appears repeatedly in its near region, the behavioral trajectory around the point may be more similar than other cases.

(iv) Moreover, the salient points are very important since these points describe the transitions between different states of stochastic behaviors. It is unwise to be averaged such as sampling [28], piecewise aggregate approximation (PAA) [29], and DTW averaging. Preservation of salient points should be considered.

The shape descriptor of trajectories takes into account of operation types, sub-trajectories, and slopes. It aims to assess the similarity of individuals' sequences or sub-sequences. Consequently, the problem of clustering is using the shape descriptor to describe the collection of segments of similar shapes in trajectories.

Definition 7. (Shape Similarity of $B T$ ) In a duration, trajectories $S_{1}$ and $S_{2}$ are similar in shape if the following three conditions hold:

(i) The operation types of nodes in trajectories are the same;

(ii) The shapes of the trajectory segments are similar. In other words, they have the same change tendency upward or downward or invariant, and;

(iii) The cumulative slope distance of segments is similar, which means the values should fall into a range of thresholds. The slope $\measuredangle$ represents the angle that a segment $s_{i}$ intersects with the positive $x$-axis, represented as

$\theta=\arctan \frac{o_{i+1} \cdot y-o_{i .} y}{t_{i+1}-t_{i}}$.

This definition firstly restrains the strict sphere of action while comparing, i.e. the comparison of two different behaviors makes no sense. Second, it specifies the major valuation strategy. And third, it estimates the relative expression degree of a behavior in terms of slope. When their time overheads are more close, the expression degrees of behaviors are more similar.

\subsubsection{Structural Similarity}

We maintain that similar behavior trajectories share a similar structural characteristics. The structure is constituted by a series of behavioral sample points, which distribute over the horizontal coordinate non-equidistantly. We model the sequence of points as a stochastic process with variability in the horizontal direction. The posterior distribution of data can be inferred with the prior knowledge. We use i.i.d zero-mean Gaussian noise model to disturb the distribution of points.

$p(x)=N\left(x \mid 0, \sigma^{2}\right)=\frac{1}{\sqrt{2 \pi} \sigma} \exp \left(-\frac{x^{2}}{2 \sigma^{2}}\right)$,

where $x$ represents the grey level [30] and $\sigma$ the standard deviation. Giving $n$ independent and identically distributed sample sequences, the conditional probability given a model parameter $\theta$ is

$p(X \mid \theta)=\prod_{i=1}^{n} p\left(x_{i} \mid 0, \sigma^{2}\right)=\left(\frac{1}{\sqrt{2 \pi} \sigma}\right)^{n} \exp \left(-\frac{1}{2} \sum_{i=1}^{n} \frac{x_{i}^{2}}{\sigma^{2}}\right)$.

Then, $\theta$ can be estimated by conjugate inverse-gamma priors as

$p(\theta)=I G(\theta \mid \alpha, \beta)=\frac{\beta^{\alpha}}{\Gamma(\alpha)} \theta^{-\alpha-1} \exp \left(\frac{-\beta}{\theta}\right)$,

where $\theta>0, \alpha$ the shape parameter, and $\beta$ the scale parameter.

Each sequence can be modeled as a probability distribution of specific symbolic elements under the condition of estimated parameters. They can be aligned by treating the sequence as a character text. Then one finds the longest common sequence using dynamic programming. Given two sequences $\operatorname{seq}_{1}=\left\{x_{1}, x_{2}, \ldots, x_{m}\right\}$ and $\operatorname{seq}_{2}=\left\{y_{1}, y_{2}, \ldots, y_{n}\right\}$, we construc$\mathrm{t}$ a two-dimensional matrix, where $M(i, j)$ records the $L C S$ 's length before $x_{i}$ and $y_{j}$, whose value is determined by positions of upper, left, and upper left. The algorithm transfers the score from the top left corner to the bottom right corner. The $L C S$ is obtained along the reverse path. The process is recursively expressed as

$M(i, j)=\left\{\begin{array}{l}0, \text { if } x_{i} y_{i}=0 ; \\ 1+M(i-1, j-1), \text { if } x_{i} y_{i} \neq 0 \text { and } x_{i}=y_{i} ; \\ \max (M(i, j-1), M(i-1, j)), \\ \quad \text { if } x_{i} y_{i} \neq 0 \text { and } x_{i} \neq y_{i} .\end{array}\right.$ 
The similarity of sequences is computed by

$\operatorname{sim}_{\text {seq }}=\left\{\begin{array}{l}0, \text { if } \min (n, m)<\varsigma \max (n, m) ; \\ \frac{M(n, m)}{\min (n, m)}, \text { otherwise. }\end{array}\right.$

The parameter $s$ is a small constant value, representing that the sequences will be neglected when the their lengths have significant difference. As for the sequences of equivalent lengths, the similarity is the ratio of the length of the common part to the length of the shorter sequence.

The structural similarity of sequences from the sequential pattern mining and biological engineering domains can be easily found in literatures [31] and [32], and we only summarize them due to the space limitation. On the one hand, we consider the elements' time overheads in LCS. Providing that the time spent on the same element in two sequences is $\lambda_{1}$ and $\lambda_{2}$, the time similarity on this element is computed by $\operatorname{sim}_{e}=\min \left(\lambda_{1}, \lambda_{2}\right) / \max \left(\lambda_{1}, \lambda_{2}\right)$. Then, the similarity of the ful$1 L C S$ is a mean of all the elements' similarities of the longest common sub-sequence ( $L C S S$ ), represented as

$\operatorname{sim}_{l c s}=\frac{1}{|L C S|} \sum_{i=1}^{|L C S|} \frac{\min \left(\lambda_{1}, \lambda_{2}\right)}{\max \left(\lambda_{1}, \lambda_{2}\right)}$.

On the other hand, LCS is the common part of sequences, which we measure the contributions to their mother sequence, i.e. measuring by weights. There are two indictors to measure the significant degree. One is the percentage of time spent, the other is the percentage of the elements in LCS. Therefore, we have

$S_{\text {structual }}(a, b)=\sqrt{\frac{\lambda_{a}}{T_{a}} \cdot \frac{|L C S|}{L_{a}}} \cdot \sqrt{\frac{\lambda_{b}}{T_{b}} \cdot \frac{|L C S|}{L_{b}}}$,

where $\lambda$ is the time cost in $L C S, T$ the time in total in the mother sequence, $L$ the length of the mother sequence, $|L C S|$ the length of $L C S$, and $a$ and $b$ the two distinctive sequences.

\subsubsection{Micro Similarity}

To satisfy condition (ii) and (iii) in Definition 7, we encode the behavioral trajectories. The aim is to transform the sequence, whose features are change tendency and segment slope, to a series of symbols. When a segment $s_{i} \in$ has a downward $(\searrow$ ) tendency, we code it -1 . Similarly, we code upward $(\nearrow$ ) tendency 1 , and invariance $(\rightarrow) 0$. Calculating the tendency can be easily achieved by the slope $k=\tan (\theta)$, i.e.

$\operatorname{code}\left(s_{i}\right)=\left\{\begin{array}{l}1, \text { if } k>0 \\ 0, \text { if } k=0 \\ -1, \text { if } k<0 .\end{array}\right.$

Furthermore, the slope is categorized into different levels according to their degrees of tilt. In terms of the extent of deviating from the horizontal direction, we define three levels significant, general, and moderate. And respectively, they are coded with Arabic numerals 3,2 and 1. Positive or negative value of the segment direction is judged by the tendency and slope, represented by their product. When the product value equals 0 , the two operations have the same expression extent and the connecting line is parallel with $x$-axis. In this way, we could use two $n$-length encoded sequences to approximately describe the trajectory. One is the tendency sequence $(T S)$ representing the up or down which contains one element in $\{-1,0,1\}$, the other is the sequence of change extent of tendency $(E S)$ which contains one element in $\{1,2,3\}$. A simple example is shown in Fig. 3.

As for the tendency sequences, we compare the same positions and determine whether they have the same codes. If yes, the counter adds by 1 , and the similarity of tendency can be computed as

$\operatorname{sim}_{\text {tendency }}=\frac{\text { count }}{|L C S|}$.

An extra benefit of coding is to make it possible to compare the slope distance of common subsequence. Given two sets of slopes $X=\left\{s_{1}, s_{2}, \ldots, s_{n}\right\}$ and $X^{\prime}=\left\{s_{1}{ }^{\prime}, s_{2}{ }^{\prime}, \ldots, s_{n}{ }^{\prime}\right\}$, the slope distance is computed as

$D_{\text {slope }}\left(X, X^{\prime}\right)=\frac{1}{6 n} \sum_{i=1}^{n}\left|c_{i} \rho_{i}-c_{i}{ }^{\prime} \rho_{i}{ }^{\prime}\right|$,

where $c \in T S, \rho \in E S$, and the figure in the denominator denotes the number of sectors partitioned by the slope codes, which depends on the meticulous degree of the partition. Here, we set it 6 . The similarity $\operatorname{sim}_{\text {slope }}\left(X, X^{\prime}\right)=1-D_{\text {slope }}\left(X, X^{\prime}\right)$, whose values fall invariably in $[0,1]$.
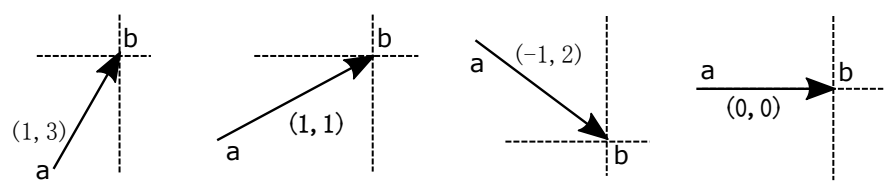

Fig. 3. A simple example of coding.

There are some basic properties of slope distance:

(i) Non-negativity: $D_{\text {slope }}\left(X, X^{\prime}\right) \geqslant 0$.

(ii) Symmetry: $D_{\text {slope }}\left(X, X^{\prime}\right)=D_{\text {slope }}\left(X^{\prime}, X\right)$.

(iii) Self-similarity: $D_{\text {slope }}(X, X)=0$.

(iv) Triangle inequality: $D_{\text {slope }}\left(X, X^{\prime}\right) \leqslant D_{\text {slope }}\left(X, X^{\prime \prime}\right)+$ $D_{\text {slope }}\left(X^{\prime}, X^{\prime \prime}\right)$.

Properties 1-3 are immediate. Now we give the proof of property 4 as follows.Proof:

$$
\begin{aligned}
\text { left } & =D_{\text {slope }}\left(X, X^{\prime \prime}\right)+D_{\text {slope }}\left(X^{\prime}, X^{\prime \prime}\right) \\
& =\frac{1}{6 n} \sum_{i=1}^{n}\left(\left|c_{i} \rho_{i}-c_{i}^{\prime \prime} \rho_{i}{ }^{\prime \prime}\right|+\left|c_{i}^{\prime \prime} \rho_{i}{ }^{\prime \prime}-c_{i}{ }^{\prime} \rho_{i}{ }^{\prime}\right|\right) \\
& \geqslant \frac{1}{6 n} \sum_{i=1}^{n}\left|c_{i} \rho_{i}-c_{i}^{\prime \prime} \rho_{i}^{\prime \prime}+c_{i}^{\prime \prime} \rho_{i}^{\prime \prime}-c_{i}{ }^{\prime} \rho_{i}{ }^{\prime}\right| \\
& =\frac{1}{6 n} \sum_{i=1}^{n}\left|c_{i} \rho_{i}-c_{i}^{\prime} \rho_{i}{ }^{\prime}\right|=\text { right. }
\end{aligned}
$$

Given a position $s_{i}$ in two aligned sequences, the similarity is related to their respective neighbors. Two positions are 
more similar if they have the same neighbors and the slopes connecting positions are more similar. We consider two cases. One is independent points that means the positions have no public neighbors, and the other is dependent points which means there are common precursor or subsequent points of the current positions within short loops. In the case of independent points, the time contribution is examined. In the case of dependent points, we further determine the similarities of trends and slopes. For example, s1 and s2 in Fig. 4 are two aligned sequences of length $6, \mathrm{C}$ and $\mathrm{B}$ in the black rectangle are dependent points because $\mathrm{C}$ has the same neighbor $\mathrm{B}$ within a (C-C) loop. While the first and second elements are independent points for they have no common neighbors.

$$
\begin{array}{ll|l|lll}
\text { s1: A B } & \text { C B } & \text { A C } & & \text { s3: C A B C D } \\
\text { s2: A D } & \text { C B } & \text { B C } & \text { s4: B A B D }
\end{array}
$$

Fig. 4. Examples. s1, s2 are two aligned sequences of length 6 and s3, s4 an example of SSS

After considered the links between data points, we further study the smallest sub-sequences ( $S S S$ ) containing LCS and concern the temporal characteristics in succession. The SSS is a sequence that embodies all the elements picking from the start position to the last position of $L C S$ in the sequence. In view of the latent sparseness of SSS, we use the $60 \%$ intensive areas of energy. There are some basic properties:

(i) SSSs have the same start and end points.

(ii) The lengths of SSSs are not necessarily equal.

(iii) The shorter one can be usually inserted with estimated values.

Suppose the LCS (ABD) is computed by dynamic programming from two sequences. Then $\mathrm{ABCD}$ and $\mathrm{ABD}$ are their respective $S S S$, like $\mathrm{s} 3$ and $\mathrm{s} 4$ in Fig. 4. A null position is inserted between $\mathrm{B}$ and $\mathrm{D}$ to make the two SSSs aligned $(A 3 S)$.

We claim that the two components (i.e. link and temporal) aforementioned have the effect of increase or decrease to micro similarity. When two behaviors occur in pairs, they and their links will be reinforced, or else they will be weakened. Therefore it makes sense to assign properly either a reward value to represent its retaining and reinforcement or a penalty value to represent its natural weakening.

The time award score (TAS) can be computed by the formula (14). Given two behavioral sample points in sequences, when the time intervals are equal, we award a user-defined maximum value, say 0.01 , else we divide 0.01 by the difference of the time intervals. The more significant the difference is, the smaller the reward one get. We set it 0 if the difference reaches a high level. The parameter $\eta$ is a very small number representing the remarkable degree of the interval lengths.

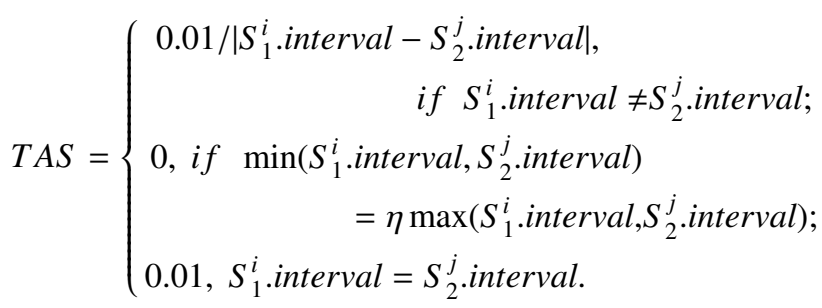

where $S_{y}^{x}$ denotes the $x^{\prime}$ th position of the $y^{\prime}$ th sequence.

Similarly, the intensity of link is quantified by the significance degree of corresponding line segment's slope as

Link $_{\text {in }}=\left\{\begin{array}{l}0.01 /\left|M S D_{1}^{i}-M S D_{2}^{j}\right|, \text { if } M S D_{1}^{i} \neq M S D_{2}^{j} ; \\ 0.01, \text { otherwise. }\end{array}\right.$

where $M S D_{y}^{x}$ denotes the mean slope distance of $x$ 'th segment in the $y$ 'th sequence.

Temporal penalty is to simulate the behavioral evolution. The behaviors are produced as time went by, and the older actions' impacts on the newer ones will fade over time. To ensure the flexibility of computation, usually we set a small dynamic changeable number.

Eventually, each sample point in the behavioral trajectory can be depicted by a 4-tuple $<T, S, \mapsto, \measuredangle>$, where $T$ represents the time interval, $S$ represents the operation type, $\mapsto \in\{-1,0,1\}$ denotes the coding of tendency, and $\measuredangle \in\{1,2,3\}$ denotes the coding of slope. The behaviors are more similar if each of the four elements is more similar. If partial elements are similar while others are dissimilar, the algorithm would adjust the TAS according to the principles of reward and punishment discussed above. The pseudo code of realizing the process is presented in Algorithm 1.

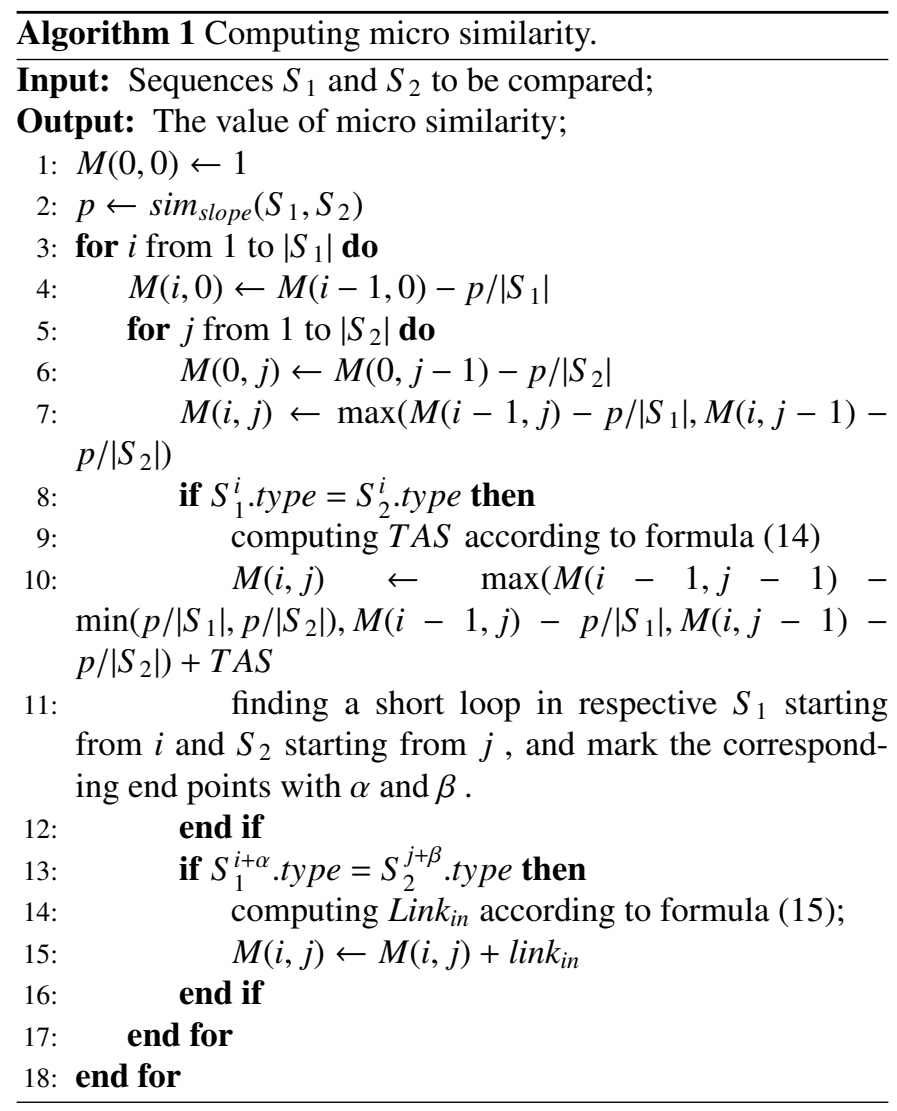

\subsubsection{Hybrid Similarity Computation}

Learning behavior is related to the inner state involved in specific learning environment, which is produced spontaneously 
from the inside out and reversely stimulates the inner procedure. When students use the system, such knowledge helps us to discover their similarities that if some of students have similar behavioral styles, their interactive actions as the external representation must share a certain similarity. The solution of comparing sequences is to compare their common parts. Then we actually used the $L C S$ twice. One was to inspect the element and time weights of the physical sequences, the other was to examine the links between the sample points in SSSs. Therefore, the final score should be a compromise of both portions. The behavioral trajectories are more similar if their general structures of the whole and micro-features of the common parts are more similar. It is simply computed as

$S_{\text {final }}(a, b)=\lambda S_{\text {structural }}(a, b)+(1-\lambda) S_{\text {micro }}(a, b)$,

where $\lambda$ is a parameter to adjust the proportion of each section. This formula tells that the structural similarity contributes $100 \times \lambda / \%$ to the final results and the remaining percentage is determined by the micro relation between data. In other word$\mathrm{s}$, when the structural similarity and micro similarity are large simultaneously, the behavioral sequences are real similar. This idea breaks through the dominant position of traditionally relying on the physical similarity. After each trajectory from a user is compared with those from its counterparts, we get a symmetric similarity matrix eventually.

\subsection{Clustering Algorithm}

The goal of clustering is to separate the collection of elements into several disjoint subsets, so that elements within a cluster are highly similar to each other while elements in different clusters have low similarity to each other. If we treat objects as the connection of vertices of a graph, the problem of clustering is equivalent to that of graph partitioning. Then students having similar behavioral styles will be automatically categorized into groups. We treat the sequences as vertices of a graph, the connections of comparing sequences as edges of the graph, and the similarities as weights of the edges. Then each sequence is a node, and each pair of nodes is connected by a weighted edge. The task is to partition the weighted graph so that the sum of weights connecting different sub-graphs is as small as possible while the sum of weights within a sub-graph is as big as possible. We choose spectral clustering rather than other clustering algorithms such as k-means and EM to achieve our goal. Spectral clustering has emerged recently as a popular clustering method which employs eigenspace decomposition of the symmetric similarity matrix. It transforms the task of clustering to that of graph segmentation by firstly constructing a weighted graph with the similarity matrix and then finding the minimum cut of the graph. The spectral clustering is superior to the counterparts primarily due to its simpler conditions, more robustness, and smaller computational complexity on our dataset. Firstly, the spectral clustering can be implemented only with the similarity matrix, which has been established from previous steps without any additional computing. Secondly, Laplacian eigenvector decomposition can capture the main features of space, and it is not sensitive to noisy data thus is expected to achieve a better clustering performance. And thirdly, eigenvectors decomposition of the matrix is a very basic operation, which is a big merit in the case of large amount of data. Given a weighted graph $G=(V, E, W)$, where $V$ is the set of vertices, $E$ the set of edges and $W$ the set of weights, the minimum cut $\operatorname{cut}(P, Q)$ is defined as partitioning $V$ into disjoint sub-graphs $P$ and $Q$ such that $\sum_{e(P, Q) \in E}^{E} w(e(P, Q)) \in W$, i.e. the sum of weights on $\{(p, q) \in E \mid p \in P, q \in Q\}$, is minimized. To segment it fairly, we use Ratio $C u t$ to minimize the following objective function.

$\operatorname{RCut}\left(c_{1}, c_{2}, \ldots c_{k}\right)=\sum_{i=1}^{k} \frac{\operatorname{cut}\left(c_{i}, \bar{c}_{i}\right)}{\left|c_{i}\right|}$

where $k$ is the number of clusters, $c$ is a label of the segmented sub-graph, the numerator represents the minimum cut between clusters, and the denominator represents the number of samples in current cluster. However, minimizing the function is a NP-hard problem that we approximate the solution by a relaxation policy. The original problem can be rewrite as $\min \operatorname{tr}\left(F^{\prime} L F\right)$ s.t. $F^{\prime} F=I$, where $\operatorname{tr}(\cdot)$ is the trace of a matrix, and $F \in R^{n \times k}$, for all $i \in\{1, \ldots, k\}$ and $j \in\{1, \ldots, n\}$, $f_{j i}=\left\{\begin{array}{l}1 / \sqrt{\left|c_{i}\right|}, \text { if } v_{j} \in c_{i} \\ 0, \text { if } v_{j} \notin c_{i}\end{array}, f_{j i} \in F, v_{j} \in V\right.$. The detailed proof is given in [33]. According to the Rayleigh-Ritz Theorem [34], the solution of the optimization problem is given by the matrix $F$ containing the top $k$ eigenvectors as columns.

There exists an edge between each pair of sequences, so we get a full graph if we connect them all. We decrease the number of edges using a dissimilar threshold so that the weight of each edge exceeds a specific value. The remaining weighted undirected graph is called a $\varepsilon^{-}$-neighborhood graph ${ }^{1}$. Given a similarity matrix $S M \in R^{n \times n}$ for $n$ sequences and a dissimilar threshold $\varepsilon$, the goal is to output the clustering results. Let $k$ be the desired number of clusters. The main steps of the algorithm is described as below.

Step 1. If $\operatorname{sim}\left(v_{i}, v_{j}\right) \geqslant \varepsilon$, connecting $v_{i}$ and $v_{j}$, and constructing a weighted undirected graph $G(V, E, W)$ and a corresponding nonnegative and symmetric similarity matrix $S M$, where $V$ is the set of vertex, $E$ the set of connecting edges, and $W$ the similarity score between nodes.

Step 2. Computing graph Laplacian matrix $L=D-N$, where $D$ is the degree matrix of $G$ and $N$ the adjacency matrix of $G$.

Step 3. Computing the top- $k$ eigenvalues and eigenvectors to form an eigenmatrix.

Step 4. Finding the best $k$ splitting points by using $k$-means.

As a result, each row class of the final matrix is the category that corresponding node of the graph belongs to. Each segmented sub-graph is the group that the corresponding students belong to.

\footnotetext{
${ }^{1}$ In most literatures, $\varepsilon$-neighborhood graph is defined as the weights of edges not exceeding $\varepsilon$. On the contrary, this paper defines it as a lower bound with a upper bound 1 .
} 


\subsection{Prediction}

Our goal in this sub-section is properly assigning a new student into corresponding group as he/she traces his/her sequences based on the moulds. We overcome it by building the students' behavioral profiles, examining active students' sequences in real-time, and making a match to a specific group. Considering that the database stores a lot of historical data and the new one comes continuously, the calculation cost is very high if each point in query data is compared with the one in moulds. We address this problem by effective dimensionality reduction while keeping most of the original features intact.

Basically, a two-phase process indexing and retrieval is schemed. Note that the coded data should not only be treated as a series of points in $x$ - and $y$-panel, but they have a certain shape and contextual information namely velocity and acceleration. The bigger slope of the line segment means a higher speed, and the larger intersection angle of the change point means a smaller acceleration. The multiple trajectories in one group share the similar geometrical and syntactic features, with which we could build a profile of the group. In view of the practical computation overhead, we reduce the times of comparison by adopting PCA to capture the principal components and filter the noisy ones. When a new query sequence is generated, it first converts to a specific form the adapter supports, and then makes a match with the moulds. The matching procedure is in essence to measure the distance between the query sequence and the moulds. We choose absolute distance function (ADF) because of its simplicity, popularity, and most importantly, benefiting from the previous disposing.

\subsubsection{Indexing: Building PCA-based Profiles}

PCA has a lot of merits as listed in literature [35]. The uppermost function maybe the dimensionality reduction and de-noising, so that the most important data can be presented clearly. The essence of PCA is the transformation of basis, making the transformed data having the maximum variance. Given that $x_{i}, 1 \leq i \leq n$, is the vector of $m$-dimensional random variables forming a $n \times m$ matrix $M$, the top-r principal components are successively selected by an orthogonal linear transformation of $M$ as the ones having the largest variance, which are the corresponding eigenvalues. Note that each element in $A 3 S$ is a 4-tuple as described. In line with the establishment of $A 3 S$ and its inherent three properties, we are able to make it simplified further so as to obtain a more compact matrix of real number ${ }^{2}$. We first lose sight of temporal interval $T$ and operation type $S$ because they are invariant on the same indexing positions of each row except the ones that inserted nulls. Then we numerically present the directions by combining the tendency with slope. Again, we use their product $\mapsto \times \measuredangle=\{0, \pm 1, \pm 2, \pm 3\}$. Next, we stuff the null positions with zeros since it's simplest, obvious and time-saved. More

\footnotetext{
${ }^{2}$ We approximate it by using the results of pair-wise alignment due to the homogeneity of sequences. More accurate operation is to align the multisequences in subgroups. However, we abandon it for the unacceptable computational complexity on our dataset.
}

complex interpolation techniques may be concerned, but we leave it in the future.

$$
M:\left(\begin{array}{ccc}
\ddots & \text { index }:<T_{i}, S_{i}> \\
\vdots & <T_{i}, S_{i}, \mapsto_{i}, \measuredangle_{i}> & \vdots \\
\ldots & \ldots & \ddots
\end{array}\right) \rightarrow M^{\prime}:\left(\begin{array}{ccc}
\ddots & \ldots & \cdot \\
\vdots & -1 & \vdots \\
\cdot & \ldots & \ddots
\end{array}\right)
$$

Fig. 5. A transformation of matrix. Suppose $\mapsto=-1$ and $\measuredangle=1$ in the $i^{\prime} t h$ observation of a vector.

Then the original complex matrix transforms to be a simple one of real number. At the same time, we add an indexing sequences at the head of columns, shown in Fig. 5. PCA is performed on the new matrix, and Singular Value Decomposition $(S V D)$ is used to extract the eigenvalues and eigenvectors.

Let $r$ be the number of principal components, which takes the vast majority of energy of the original matrix. We compute the cumulative energy ratio no less than a cutoff of $95 \%$ to determine $r$.

$$
r=\arg \max _{r}\left(\frac{\sum_{1}^{r} \lambda_{i}}{\sum_{1}^{n} \lambda_{i}} \times 100 \%\right) \geqslant 95 \%,
$$

where $\lambda$ denotes the eigenvalue. The $r$ principal components are the profiles of the cluster, because they approximately capture most of the features. The new indexing structure is a $n \times r$ dimensional projective matrix $M^{\prime \prime}$.

\subsubsection{Retrieval: Matching a Sequence with the Moulds}

In the retrieval phase, we concern the new active users' behavioral sequence $(Q S)$ and choose some of its salient points as the query data. The salient points are the ones with high speeds $s p$ and large angles an. They are defined as

$$
\left\{\begin{array}{l}
s p_{p_{i}}=\frac{\left\|p_{i}-p_{i+1}\right\|}{\Delta t} ; \\
a n_{p_{i}}=\cos ^{-1} \frac{\left\|p_{i}-p_{i-1}\right\|^{2}+\left\|p_{i}-p_{i+1}\right\|^{2}-\left\|p_{i-1}-p_{i+1}\right\|^{2}}{2\left\|p_{i}-p_{i-1}\right\| \cdot\left\|p_{i}-p_{i+1}\right\|} .
\end{array}\right.
$$

where $p$ denotes the point vector, $\|\cdot\|$ the norm of the vector, and $\Delta t$ the time interval of two points. The $p_{i}$ is retained if $a n_{p_{i}}$ and $s p_{p_{i}}$ respectively exceed a certain thresholds, say $2 \pi / 3$ and 0.5 . Finally, we get a new query sequence $Q S^{+}$containing all the desired salient points. We use the indexing sequence $Q I S$ as its head and the sequence of real number $Q N S$ as its factual computational section. Three major steps are provided to get the distance between $Q S^{+}$and distinctive $M^{\prime \prime}$ s.

Step 1. Finding the set of all positions $P$ in the head sequence of $M^{\prime \prime}$ that have the same types with the elements in QIS .

Step 2. Computing the penalty weight $w$ on each position as

$$
w_{l}^{q}=\frac{\left|\mu_{l} \cdot T-\tilde{\mu}_{q} \cdot T\right|}{\max \left(\mu_{l} \cdot T, \tilde{\mu}_{q} \cdot T\right)}
$$

where $q$ denotes the order number in $Q S^{+}, l$ the order number in $P, l \leqslant|P|-1$. E.g. $\tilde{\mu}_{q}$ is the $q$ 'th querying position in $Q S^{+}$. 
Step 3. Computing the mean cumulative distance as

$$
\left\{\begin{array}{l}
D\left(Q S^{+}, M^{\prime \prime}\right)=\frac{1}{n} \cdot \sum_{i=0}^{n-1}\left(\sum_{q \in Q S^{+}, l \in P}^{\min (v, r)} w_{l}^{q} \cdot d\left(\tilde{\mu}_{q}, u_{l}\right)^{2}\right)^{\frac{1}{2}} ; \\
d\left(\tilde{u}_{q}, u_{l}\right)=\mid \tilde{u}_{q} . \text { value }-u_{l} \cdot \text { value } \mid .
\end{array}\right.
$$

where $v=\left|Q S^{+}\right|$, and $r$ the number of principal components. If there is no match between positions in QIS and those in the $i$ 'th row of $M^{\prime \prime}, d(\cdot, \cdot)=0$. The distance of positions is calculated by ADF. Consequently, we classify $Q S$ into the cluster that has the smallest distance.

\section{Evaluations}

To conduct the experiments, we collect a real dataset and design a synthetic data generator to evaluate the proposed framework. The real dataset is used for building the similarity models and grouping students. The synthetic data generator is used to test the on-line prediction ability. All the experiments are scripted either by Java or by Matlab, performed on a $2.20 \mathrm{GHz}$ machine with 4GB memory and Windows 7 64bit system.

\subsection{Data Description}

The real dataset was collected from SkyClass in distance learning school of Xi'an Jiaotong University. SkyClass is a platform supporting live video and VOD, which is composed of PCs and smart phone clients. We collect on-line students' interactive $\log$ s from Sep. 1st, 2014 to Jan. 10th, 2015, totally 130 days. We obtained 14,002,772 behavioral sample points from 57,717 students. After some preprocessing such as data cleaning, feature selection and column-row switching, we use the students' ID and date as the key, and finally get 518,532 sequences. A student's sequences may cross multiple days. The minimum day is 1 , the maximum 125 , and averagely 12 . The students' active performances have significant difference. There are 2252 students whose cumulative days are 10, while 6,100 students stay at the lowest level (i.e. 1 day). Besides, the lengths of sequences vary from person to person. Most of them are short, while others are long with the maximum 993 and average 242. The long sequences comprise more operations thus are more valuable and should be reserved. As for the short ones, we adopt a simple cut-off strategy, which has at least two advantages: reducing the computation overhead, and obtaining a more stable result. Others' work [36] has revealed that there is a power law distribution between the lengths of sequences and the number of students. In other words, the students decrease exponentially as the lengths increase and most of students are intensively distributed over the area of short sequences. Therefore, we choose the third quartile of students, which corresponds to sequences of length around 200. In addition, in view of the right-skewed probability density curve of sequences, the median is less than the mean also verifies our choice. To ensure an effective analysis, a lower bound 200 is used as the cut-off criteria. It makes the data more meaningful and space more reduced.
The learning behaviors are the real-time increasing streams. To simulate this dynamic in the phase of prediction, we design a synthetic data generator. Table 1 lists the main parameters and related explanations. Initially, the generator generates Cos null behavioral sequences $S:<s_{1}, s_{2}, \ldots, s_{C o n}>$ and corresponding time sequences $T:<t_{1}, t_{2}, \ldots, t_{C o n}>$. For each $s_{i}, 1 \leqslant i \leqslant C o n$ , selecting randomly a probability scheme theme $e_{j} \in$ Theme, $1 \leqslant j \leqslant \mid$ Theme $\mid$. For each element in Set s.t. theme $_{j}$, it generates a behavior $b_{u} \in S$ et every Rgt interval, and appends the sequences by $s_{i} \leftarrow s_{i}+b_{u}, t_{i} \leftarrow t_{i}+R g t$.

Table 1: Main parameters and related explanations of the generator.

\begin{tabular}{|c|c|c|}
\hline Parameter & Alias & Function \\
\hline Theme & Group programs. & $\begin{array}{l}\text { To store the probability dis- } \\
\text { tribution of groups. }\end{array}$ \\
\hline Con & $\begin{array}{l}\text { Amount of concur- } \\
\text { rency. }\end{array}$ & $\begin{array}{l}\text { To simulate the on-line ac- } \\
\text { tive students. }\end{array}$ \\
\hline Set & $\begin{array}{l}\text { Symbolic set of op- } \\
\text { eration types. }\end{array}$ & $\begin{array}{l}\text { To simulate the behavioral } \\
\text { types. }\end{array}$ \\
\hline$R s s$ & $\begin{array}{l}\text { Randomly selecting } \\
\text { a scheme. }\end{array}$ & $\begin{array}{l}\text { To simulate the behavioral } \\
\text { style of a group. }\end{array}$ \\
\hline$R g t$ & $\begin{array}{l}\text { Randomly generat- } \\
\text { ing a time amount. }\end{array}$ & $\begin{array}{l}\text { To simulate the operational } \\
\text { interval. }\end{array}$ \\
\hline
\end{tabular}

\subsection{Evaluation Method}

The experiment comprises three parts. The first part focuses on the similarity of behavioral sequences. We compare the differences between our method and others. First, we consider the physical characteristics of sequences only, then we gradually take other dimensions into consideration. Concretely, we compare:

(i) Element-only $(E)$ : Only consider the element contribution.

(ii) Temporal-only $(T)$ : Only consider the time contribution.

(iii) Structure-only $(S)$ : Consider both the element and time contributions. It is adopted by Lettner et al.(2014) and Chen et al. (2011).

(iv) Micro-only $(M)$ : Only consider the micro dimension.

Our method Hybrid is abbreviated as $H$, which incorporates both the structural and micro dimensions. We model the evaluation as an information retrieval process as follows.

Each cell in the matrices is viewed as a relevance score. We rate the cell value in the light of the rules shown in Table 2. A five rating scheme represents the similarity levels. It is worth noting that the division intervals are not equidistant, because we would like to ensure a broader section for the similar case. Figuratively, it is something like an inverted pyramid format, where each layer spans $2^{l-1}, 1 \leqslant l \leqslant 3$, using 0.1 as the unit. The consideration is that the longer the sequences are, the lower probability their similarities fall in the higher divisions. When it is rated 4, it means the students are highly similar; when it is rated 0 , however, it means the students are absolutely dissimilar at the bottom of the pyramid. We pick up 20 sequences as the queries. The MAP and $n D C G$ are calculated for one retrieval. Finally, we calculate the mean MAP and $n D C G$ of all the retrievals. 
Table 2: A five rating scheme.

\begin{tabular}{llll}
\hline Rating & Meaning & Division & Interpretation \\
\hline 4 & $\begin{array}{l}\text { Strongly } \\
\text { similar }\end{array}$ & {$[0.8,1]$} & Students are highly relevant. \\
3 & $\begin{array}{l}\text { Very } \\
\text { similar }\end{array}$ & {$[0.4,0.8)$} & $\begin{array}{l}\text { Students are of very similar } \\
\text { behavioral styles. }\end{array}$ \\
2 & $\begin{array}{l}\text { Similar } \\
\text { Not very } \\
\text { similar } \\
\text { Not simi- } \\
\text { lar }\end{array}$ & {$[0.2,0.4)$} & $\begin{array}{l}\text { The relevance is moderate. } \\
\text { The relevance is weak. }\end{array}$ \\
& {$[0.1)$} & $\begin{array}{l}\text { Students are absolutely irrele- } \\
\text { vant. }\end{array}$ \\
\hline
\end{tabular}

$M A P$ denotes the mean average precision literally, which is the most frequently used measure of ranked problem in the information retrieval field. In our experiment, it stands for the mean average similarity rating after each querying sequence is retrieved. Because the group members should be similar to each other, we consider the case whose rating is greater than or equal to 2 . The value is computed by

$M A P=\frac{\sum_{k=1}^{N} P(k) \times \operatorname{rel}(k)}{\text { number of relavant sequences }}$,

where $N$ is the number retrieved, $k$ the $\operatorname{rank}, \operatorname{rel}(\cdot)$ the binary function of the relevance of a given rank, and $P(\cdot)$ the precision of a rank with a cut-off, computed by

$P(k)=\frac{\mid \text { relevant value of rank } k \mid}{k}$.

$n D C G$ (Normalized Discounted Cumulative Gain) is used further to consider the relevant levels of sequences. It is calculated as

$n D C G[i]=Z_{n} \sum_{i=1}^{n}\left(2^{r(i)}-1\right) / \log (1+i)$,

where $i$ is the position index and $Z_{n}$ the normalization calculated by $D C G[i] / \max D C G$.

The second part of the experiment is to study the results of spectral clustering. The selection of $k$ greatly influences the grouping quality. Therefore we have an aim to select the best $k$ to make a relatively reasonable group distribution. Besides, we also study the parameters under different conditions. We evaluate the relative quality of grouping by giving a certain parameters and varying $k$. Hence, we borrow the idea of Normalized Mutual Information (NMI), which can be equivalently expressed as $N M I(X, Y)=\frac{2 I(X ; Y)}{H(X)+H(Y)}$, where $I(\cdot ; \cdot)$ is mutual information, and $H(\cdot)$ the entropy. However, the difference to the standard NMI is that the standard NMI is used to measure the gap of clustering results and the actual ones. In this experimen$t$, it is difficult to label the data sequences in advance, which disables the evaluation of clustering quality. Instead, we adopt a cross validated $N M I$, i.e. we compute the mean of $N M I$ after pair-wise comparing the result on specific $k$ with the ones on other values excluding $k$. The $k$ is selected from 2 to 10 , therefore we conduct 9 folds. It stands for the stability degree of a scheme compared to others.
Ideal grouping makes each group have a comparable amount of members, which is actually difficult to meet. We calculate the relative size of groups to further decide a proper scheme. Given a grouping scheme $i$, the minimum size of groups $p_{\min }(i)$, and maximum $p_{\max }(i)$, we consider the relative size in extreme cases $p(i)=p_{\min }(i) / p_{\max }(i)$. This value falls between 0 and 1 . The bigger the value is the more close of group size is. Conversely, it denotes at least one of the groups is too big or too small.

The last part of the experiment aims to examine the performance of prediction. The essence of prediction is also a problem of similarity measurement. The shorter distance between a sequence and the moulds, the higher similarity it is and thus one should packet the sequence into the group with the lowest score. We compare our approach with two baselines. The first one is computing by Tanimoto Distance (TD), an extended for$\mathrm{m}$ of Jaccard Distance. And the second one is computing by Cosine Distance $(C D)$. Because $s$ and $M$ can be viewed as short documents. We use term frequency $(T F)$ to build the Vector Space Models (VSM) and further run the baselines. Given a sequence $s$ and an $n$-dimensional matrix $M$, the mean cumulative Tanimoto Distance is computed by

$T D(s, M)=\frac{1}{n} \sum_{i=1}^{n} \frac{s \cdot M[i]}{\|s\|^{2}+\|M[i]\|^{2}-s \cdot M[i]}$,

and the mean cumulative Cosine Distance is computed by

$C D(s, M)=\frac{1}{n} \sum_{i=1}^{n} \frac{s \cdot M[i]}{\|s\| \times\|M[i]\|}$,

where $M[i]$ denotes the $i^{\prime}$ th row of matrix, ". " the dot product, and " $\| "$ the norm.

The evaluation metric is mean accuracy $(M A)$. When the generator produces a scheme randomly, we record its corresponding group number and compare it with the grouping result. A grouping is correct if they are consistent, or else it is incorrect. The accuracy is quantified as the ratio of correct groupings to the total.

\subsection{Results Analysis}

The MAP among different approaches under condition $\lambda=0.5$ is shown in Fig. 6. Clearly, $E$ gets the highest value 1.0 , followed by $T$. However, almost all the literatures did not adopt them because they focus on the contribution of a single component regardless of others. For example, $E$ only considers the element's contribution without the temporal information and $T$ vice versa. $S$ is a widely used method, where we only get 0.28 despite of the high scores of $E$ and $T$. This is because $S$ directly degrades the similarity of sequences by combining $E$ and $T$. When $M$ is considered only, we get 0.504 offset. $M$ is a remedy of $S$ not considering the data interaction and link model. Consequently, we get $52.1 \%$ promotion compared to the $S$ method. Fig. 7 further differentiates the hybrid approach from others using $n D C G$ evaluation. The result is similar to $M A P$, and we get $53.3 \%$ promotion compared to $S$. 


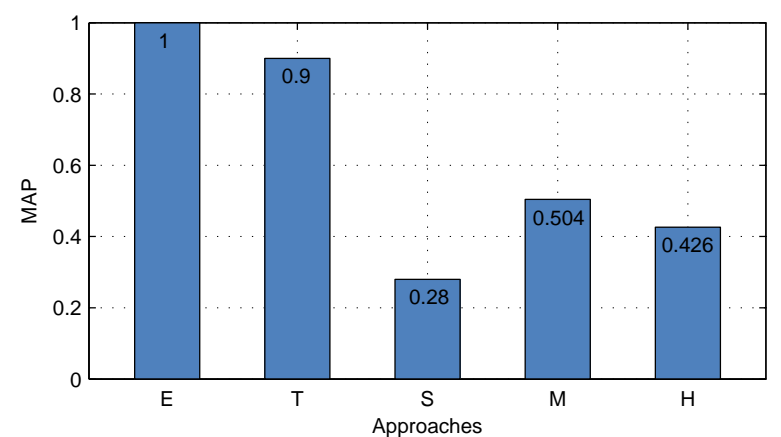

Fig. 6. $M A P$ among approaches.

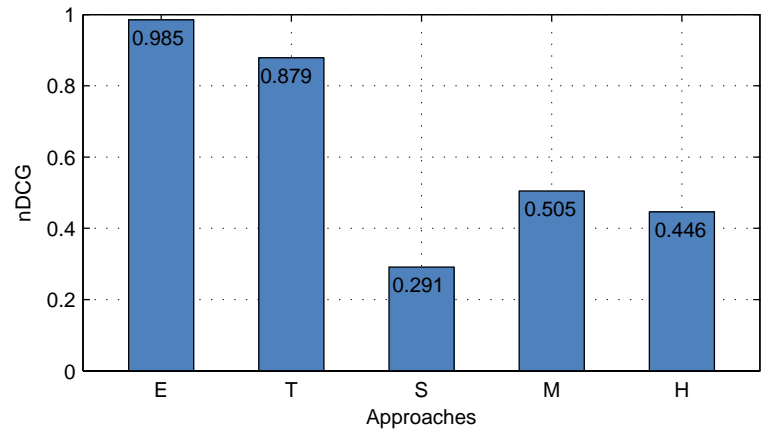

Fig. 7. $n D C G$ among approaches.

Fig. 8 shows the impact of $\varepsilon^{-}$valuing from 0 to 0.6 on $M A P$ and $n D C G$ when $\lambda=0.5$. Overall, the MAP outnumbers $n D$ $C G$ when $\varepsilon^{-}>0.1$. There is little significant change of $M A P$ when $\varepsilon^{-}<0.2$, then a peek occurs when $\varepsilon^{-}=0.3$. The MAP decreases dramatically when $\varepsilon^{-}>0.5$. The similar trend happens to $n D C G$. The difference is that it decreases rather than slightly increases when $\varepsilon^{-}<0.2$. This figure implies that $M A P$ and $n D C G$ change as $\varepsilon^{-}$changes. $\varepsilon^{-}$is a filter that the smaller it is the more complete the data will be. It does not mean one will get the higher MAP and $n D C G$ with a smaller $\varepsilon^{-}$. In our dataset, $\varepsilon^{-}$around 0.3 is an optimal choice.

The impact of $\lambda$ on MAP and $n D C G$ when $\varepsilon^{-}=0.3$ is shown in Fig. 9. It shows the $M A P$ and $n D C G$ are mutual winding. The value at each position is close. The trends of lines first raise fast from 0.1 to 0.55 when $\lambda<0.2$. Then they hold summit until $\lambda=0.4$. Afterwards, they decrease smoothly from the summit to bellow $0.3(0.4<\lambda<0.8)$. This result indicates that we could select an optimal $\lambda$ to get higher $M A P$ and $n D C G$.

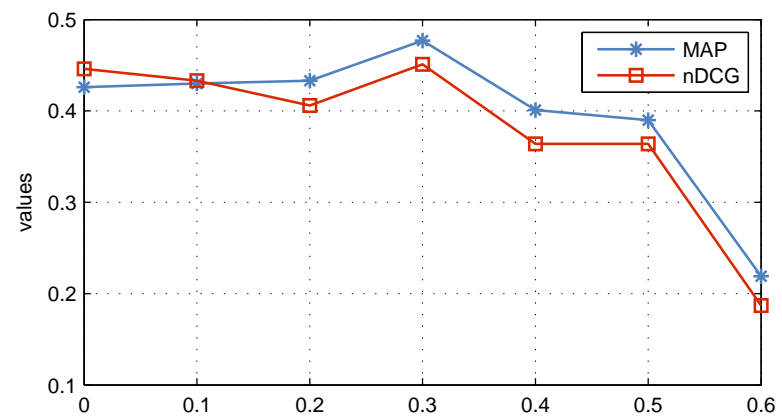

Fig. 8. The values of $M A P$ and $n D C G$ when $\lambda=0.5$ and varying $\varepsilon^{-}$from 0 to 0.6 .

The parameter $k$ denotes the number of groups. Fig. 10 shows $N M I$ varying with $k$. The $N M I$ is very low at first $(k=2)$, then it increases linearly until $k=5$. After that, $N M I$ reaches a small peak for the first time $(k=6)$, then it decreases and rebounds when $k=7$. NMI reaches the second peak when $k=9$. This means if we divide the students into 6 or 9 groups, we will get a relatively stable grouping. However, the relative size $p(k=6)=0.781$ and $p(k=9)=0.102$, which indicates $k=6$ is an optimal choice.

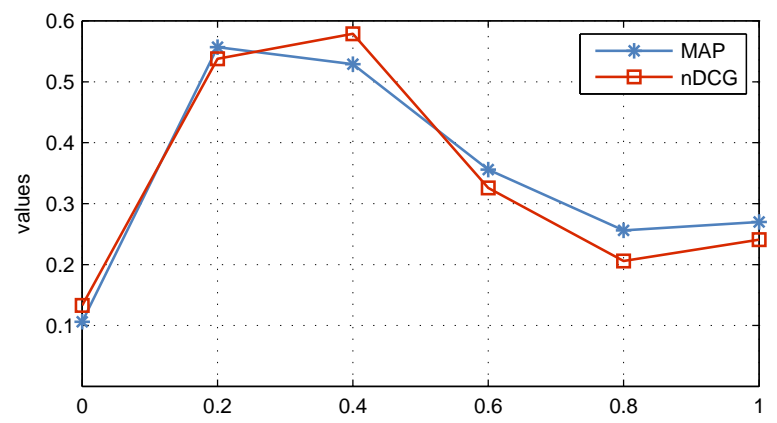

Fig. 9. The values of $M A P$ and $n D C G$ when $\varepsilon^{-}=0.3$ and varying $\lambda$ from 0 to 1 .

Fig. 11 and Fig. 12 show two different sides of the parameters' impacts on clustering results. In Fig.11, the NMI increases significantly as $k$ increases from 2 to 4 , then the trend lines almost remain steady in a relatively broader interval $(4<k<8)$. The lines have downward trends when $k$ exceeds 8 . Vertically, a clear hierarchy is presented as $\varepsilon^{-}$varies from 0.1 to 0.6 . The smaller the $\varepsilon^{-}$is, the topper layer the line is positioned. However, the case of $\varepsilon^{-}=0.2$ outperforms the one of $\varepsilon^{-}=0.1$ and the line is flatter especially when the grouping number is small. Fig. 12 tells the same thing, and the only difference is the newly defined visual dimension. It shows how different the NMI is under the condition of distinctive $k \mathrm{~s}$. The NMIs vary considerably when $k=2$ and 3 , but they assemble more closely as $k$ is larger than 4 .

Fig. 13 compares the differences before and after grouping. The first line describes an arrangement of sequences in $x$ - and $y$ - panel that come from the raw data. The second line (from Group Three) and the third line (from Group Five) are the results under the conditions of the determined parameters. All the data samples randomly and are differentiated by colors. We learn that the mixed data before grouping becomes more formatted after grouping. For example, the central part of the second line indicates the corresponding students are of very similar behavioral performance, while the remaining has a clear structure. The third line has another form of arrangement that they are more compact and somewhat matched rivals. Each subfigure displays 25 sequences for a better vision. But the finding doesn't vary with selection of samples in that as they are changed, this phenomenon maintains.

A second comparison is shown in Fig. 14. Again, we respectively adopt random sampling from the raw data and Group Three. Each color block in the figure represents the degree of correlation between students. A higher color value represents a closer degree, and the value approximating to 0 means the least relevance. On the left for example, the students 
from line 6 are very similar to others while the students in column 17 and line 15 are not similar to almost all. It's very clear that the distribution is more sparse on the left, and most dissimilar cases take up substantial space and the opposite extreme ones also catch the eye. In other words, all kinds of cases (similar, dissimilar and betweenness) are lumped together that the students are scattered and not separated on the left while the students are relatively homogeneous after grouping on the right. It also shows that there is a small portion of cases having the lowest value after grouping. This is largely because some edges, due to the threshold, might not formed between all nodes in the graph partitioning but they grouped by the transfer of relations.

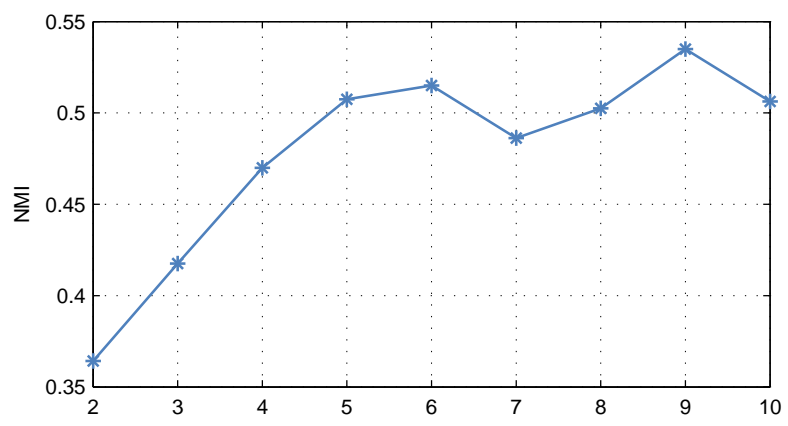

Fig. 10. $N M I$ under different $k$.

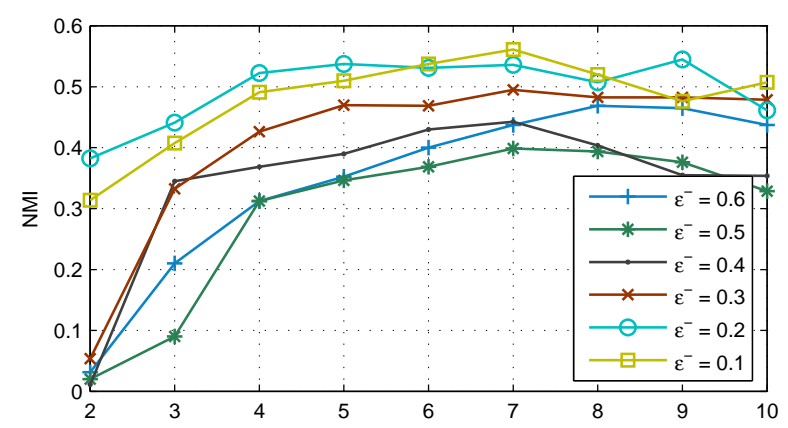

Fig. 11. $N M I$ under a certain $k$ and different $\varepsilon^{-}$

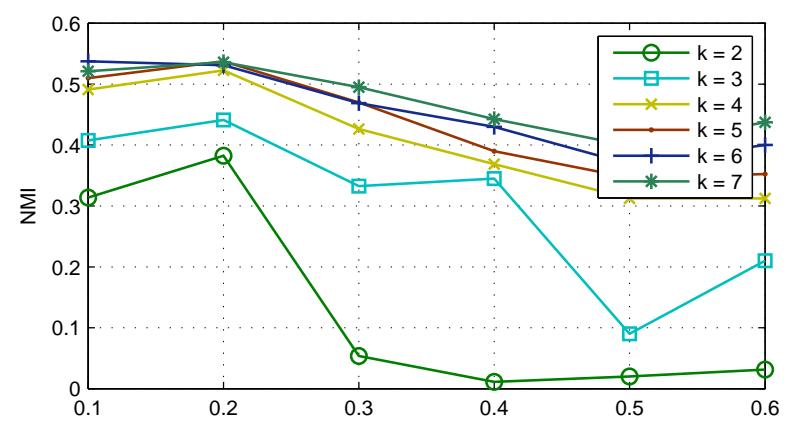

Fig. 12. $N M I$ under a certain $\varepsilon^{-}$and different $k$.

In prediction, Fig. 15 describes the comparison results between our method and baselines in terms of $M A$ with the amount of concurrency increasing. We learn that the prediction ability of Tanimoto measure is the worst. By contrast, Cosine measure seems to perform better. And our method is superior to the Cosine measure. The factors that cause this phe- nomenon, in our opinion, may mainly attribute to two aspects. First, our method processed by carefully selecting the salient points, which scaled down the scope of search, amplified the homogeneity, and thus improved the accuracy. And second, Tanimoto and Cosine measures did not consider added information except the VSM, which confused the importance among the operation types of sequences. Back to the figure, the $M A$ in our method is always higher than 0.93 . While the $M A$ in Cosine measure is around 0.9 , and the $M A$ in Tanimoto measure is often below 0.8 . These data also suggests that the amount of concurrency has little influence on $M A$.

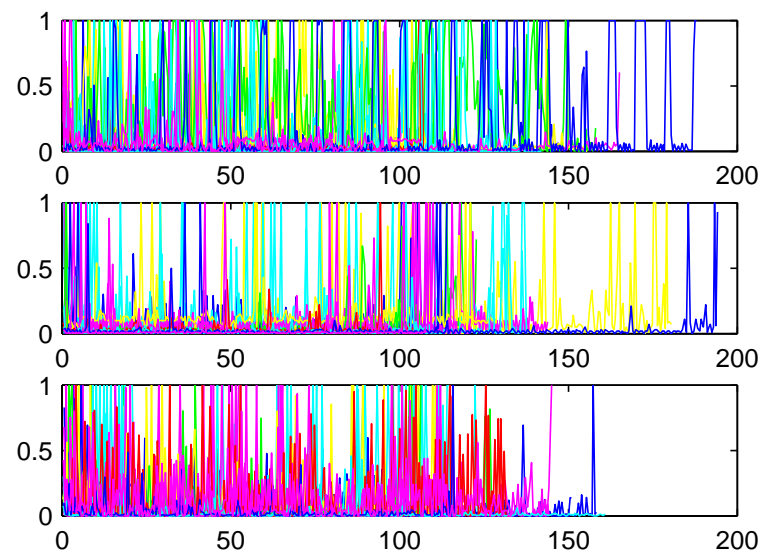

Fig. 13. Comparison I. The first line shows an arrangement before grouping, and the other two are the results after grouping.
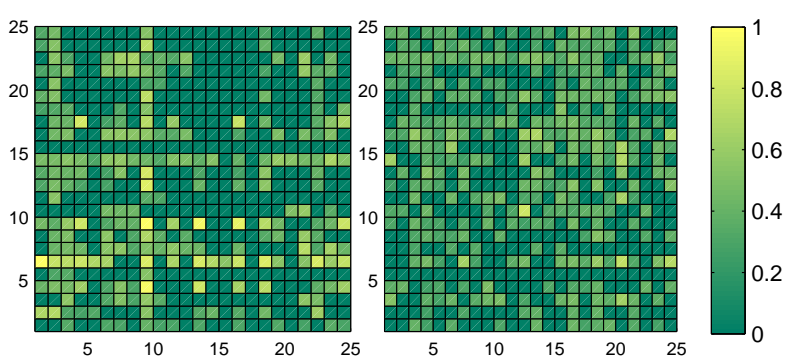

Fig. 14. Comparison II. The left hand depicts the students' distribution before grouping, and the right hand presents the case of after grouping.

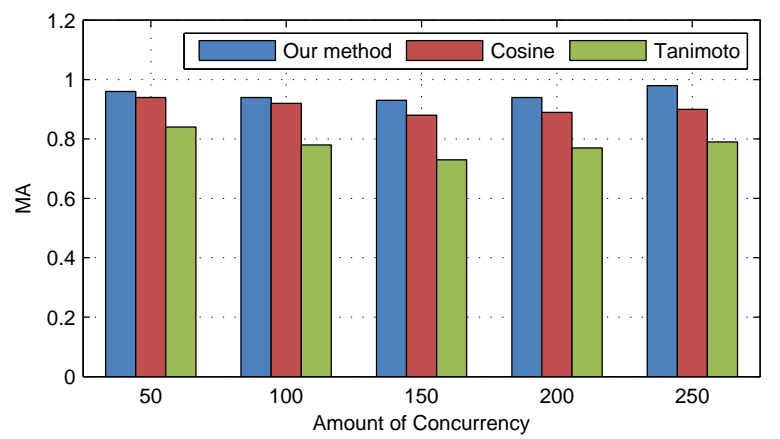

Fig. 15. Comparison between our method and the baselines in terms of $M A$ as the amount of concurrency increases.

The comparison between our method and the baselines in terms of $M A$ as the length of $Q S$ increases is shown in Fig. 16. The prediction abilities are weak as length $=25$ and the $M A$ increases as the length increases. Overall, our method outperforms the baselines. The $M A$ is related to the length of 
$Q S$, but the values rapidly tend to be stable.

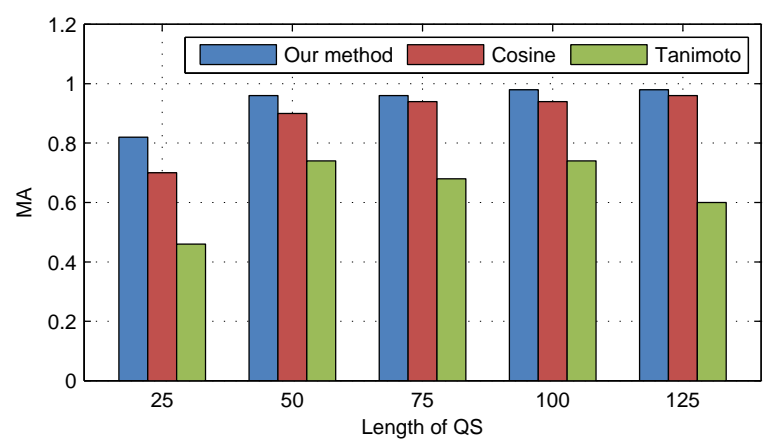

Fig. 16. Comparison between our method and the baselines in terms of $M A$ as the length of $Q S$ increases.

\subsection{Discussion}

The sequences across days from a student are of homogeneity, either the lengths are equivalent or the types are similar. We consider this case while designing the rating scheme. After running the experiment, they are separated nicely. The similarity of sequences from an individual gets a very high score than those from inter individuals, and the length of $L C S$ is much longer as well. By contrast, the sequences from diverse students are of lower similarity. This discovery verifies the initial hypothesis that a sequence exclusively represents a student's learning process and we could group students according to the similarities of their behavioral sequences.

Because of diversities of students' enthusiasms, the number of behavioral outputs makes significant difference in unit time. The positive students' online days are much more than the nonpositive. If we use the student's ID as the key, we will get many similarities 0 s according to the equation (8). In order to address this problem, we use the date as the key. Then it often compares sequences from two students with multiple times. However, it doesn't affect the final results very much because we isolate the sequences of self-similarity at the top of the pyramid in Table 2. The value equals $\sum_{i=1}^{n} S i m_{i} / n$, where $n$ is the comparing times and Sim the similarity. In this experiment, 200 as a threshold is treated as a qualified boundary to discriminate the positive and non-positive sequences, which filters most of the unqualified ones and leaves what we are greatly interested in. Then the computational overhead reduces to an acceptable level. However, the value is not fixed depending on the purpose of the experiment and other global considerations.

Now, we have basically solved the initial problem whether we could process grouping for a more homogenous group structure. The homogenous sequences are clustered in the same group. A group represents a class of students with the similar behavioral styles, which reflect alike learning processes. However, the major limitation lies in the high computational complexity in the off-line phase. Currently, accurate comparison between the sequences consumes many memories, and efficient method is still an open question. Given $n$ sequences with average length $m$ in database, the algorithm would traverse every sequences and every sample points, and the computational com- plexity is $O\left(n^{2} m^{2}\right)$. For example, the pair-wise comparisons of sequences in this experiment are $C_{2}^{2871} \approx 4.12 \times 10^{6}$, not to mention the length of the sequence. A major strategy of reducing the computation is to narrow down the data size. So it is necessary to build a condensed model for relieving the stress in the on-line phase, where fast grouping is propitious to understand students and provide an important basis for the teacher to tailor a proper teaching plan.

\section{Conclusion and Further Study}

In this paper, we have studied the behavioral sequences of students in an e-learning system and proposed a novel framework for grouping students and predicting which group a new student belonged to. The framework is phased into two. The phase I includes three major steps: 1) a new data model is described; 2) a new approach to compare students' similarities is schemed, and; 3) a sequential clustering algorithm for partitioning the graph is extended based on the spectral clustering. In phase II, we designed an on-line prediction mechanism, so as to classify a new querying sequence into the corresponding group instantly.

To examine the availability of proposed framework, we conducted a series of experiments. First, we compared the difference between the proposed similarity measure and the existed approaches using two indicators from information retrieval domain. Second, we studied the impact of different parameters on the grouping results, aiming to obtain optimal grouping results with selected conditions. And third, we studied the performance of proposed prediction strategy using two baselines. In summary, the proposed framework looks friendly to handle the problems of modeling the off-line sequences and predicting on-line. This technique has some significant merits in comparison with the existed methods. Most importantly, it considers the underlying senses of behaviors and makes grouping more meaningful.

The future work to be undertaken includes visualization of grouping results and presenting a case study on the results for a further examination. It is not an easy job to effectively measure the quality of grouping digitally unless we put it into the real learning conditions. Our tentative idea is to recruit some volunteers to work in different grouping modes, and we compare the gaps among approaches. This consideration, however, may lead to another research scope i.e. learning analysis. As for the mining task itself, we would progressively refine the corresponding procedures that have been simplified for the moment, such as the aforementioned interpolation technology and the selection of valid behaviors. All things being equal,we would use the optimal grouping scheme to mark a reasonable boundary for personalization, so that it clearly presents the heterogeneity and homogeneity between and within groups, which is capable of providing evidence for the distribution of personalized learning programs. Then together with the analysis of individual's behavioral patterns, we would like to ultimately realize a novel personalized recommender system. 


\section{References}

[1] E. Szewkis, M. Nussbaum, T. Rosen, J. Abalos, F. Denardin, D. Caballero, A. Tagle, C. Alcoholado, Collaboration within large groups in the classroom, Int. J. Comput. Collab. Learn. 6 (2011) 561-575.

[2] S. Glen, Educating for interprofessional collaboration: teaching about values, Nurs. Ethics. 6 (1999) 202-213.

[3] D.S. Stein, C.E. Wanstreet, J. Calvin, C. Overtoom, J.E. Wheaton, Bridging the transactional distance gap in online learning environments, Am. J. Distance Educ. 19 (2005) 105-118.

[4] L. Talavera, E. Gaudioso, Mining student data to characterize similar behavior groups in unstructured collaboration spaces, in: Work. Artif. Intell. CSCL. 16th Eur. Conf. Artif. Intell., 2004: pp. 17-23.

[5] J. Moreno, D.A. Ovalle, R.M. Vicari, A genetic algorithm approach for group formation in collaborative learning considering multiple student characteristics, Comput. Educ. 58 (2012) 560-569.

[6] A. Zapata, V.H. Menndez, M.E. Prieto, C. Romero, Evaluation and selection of group recommendation strategies for collaborative searching of learning objects, Int. J. Hum. Comput. Stud. 76 (2015) 2239.

[7] A.G. Hilliard, Behavioral style, culture, and teaching and learning, J. Negro Educ. 61 (1992) 370-377.

[8] D. Perera, J. Kay, I. Koprinska, K. Yacef, O.R. Zaane, Clustering and sequential pattern mining of online collaborative learning data, IEEE Trans. Knowl. Data Eng. 21 (2009) 759-772.

[9] Y. Ye, Y. Zheng, Y. Chen, J. Feng, X. Xie, Mining individual life pattern based on location history, in: 2009 Tenth Int. Conf. Mob. Data Manag. Syst. Serv. Middlew., IEEE, 2009: pp. 1-10.

[10] D. Wu, J. Lu, G. Zhang, A fuzzy tree matching-based personalized e-learning recommender system, IEEE Trans. Fuzzy Syst. 23 (2015) 24122426.

[11] J. Chen, X. Li, W.K. Cheung, K. Li, Effective successive POI recommendation inferred with individual behavior and group preference, Neurocomputing. (2016)

[12] J. van der Laan Smith, R.M. Spindle, The impact of group formation in a cooperative learning environment, J. Account. Educ. 25 (2007) 153-167.

[13] R. Seethamraju, M. Borman, Influence of group formation choices on academic performance, Assess. Eval. High. Educ. 34 (2009) 31-40.

[14] P. Blatchford, E. Baines, P. Kutnick, C. Martin, Classroom contexts: Connections between class size and within class grouping, Br. J. Educ. Psychol. 71 (2001) 283-302.

[15] C.-M. Chen, C.-Y. Li, T.-Y. Chan, B.-S. Jong, T.-W. Lin, Diagnosis of students online learning portfolios, in: 2007 37th Annu. Front. Educ. Conf. Eng. Knowl. Without Borders, Oppor. Without Passports, IEEE, 2007: pp. 17-22.

[16] A. Ounnas, H. Davis, D. Millard, Semantic Web-based Group Formation for E-learning, (2008).

[17] S. Graf, R. Bekele, Forming heterogeneous groups for intelligent collaborative learning systems with ant colony optimization, in: Int. Conf. Intell. Tutoring Syst., Springer, 2006: pp. 217-226.

[18] M. Cocea, G.D. Magoulas, User behaviour-driven group formation through case-based reasoning and clustering, Expert Syst. Appl. 39 (2012) 8756-8768.

[19] L. Shi, A.I. Cristea, M.S.K. Awan, M. Hendrix, C. Stewart, Towards understanding learning behavior patterns in social adaptive personalized elearning systems, in: Proc. 19th Am. Conf. Inf. Syst. (AMCIS 2013), AMCIS, 2013.

[20] D. Pai, A. Sharang, M.M. Yadagiri, S. Agrawal, Modelling visit similarity using click-stream data: A supervised approach, in: Int. Conf. Web Inf. Syst. Eng., Springer, 2014: pp. 135-145.

[21] G. Poornalatha, S.R. Prakash, Web sessions clustering using hybrid sequence alignment measure (HSAM), Soc. Netw. Anal. Min. 3 (2013) 257 268.

[22] S.E. Schaeffer, Graph clustering, Comput. Sci. Rev. 1 (2007) 27-64

[23] M. Casarrubea, G.K. Jonsson, F. Faulisi, F. Sorbera, G. Di Giovanni, A. Benigno, G. Crescimanno, M.S. Magnusson, T-pattern analysis for the study of temporal structure of animal and human behavior: a comprehensive review, J. Neurosci. Methods. 239 (2015) 34-46.

[24] K. Lewin, Principles of topological psychology, Read Books Ltd, 2013.

[25] T. Fu, A review on time series data mining, Eng. Appl. Artif. Intell. 24 (2011) 164-181.

[26] W. Meesrikamolkul, V. Niennattrakul, C.A. Ratanamahatana, Shape- based clustering for time series data, in: Pacific-Asia Conf. Knowl. Discov. Data Min., Springer, 2012: pp. 530-541.

[27] S.-W. Kim, J. Yoon, S. Park, T.-H. Kim, Shape-based retrieval of similar subsequences in time-series databases, in: Proc. 2002 ACM Symp. Appl. Comput., ACM, 2002: pp. 438-445.

[28] K.J. strm, On the choice of sampling rates in parametric identification of time series, Inf. Sci. (Ny). 1 (1969) 273-278.

[29] E. Keogh, S. Chu, M. Pazzani, Ensemble-index: A new approach to indexing large databases, in: Proc. Seventh ACM SIGKDD Int. Conf. Knowl. Discov. Data Min., ACM, 2001: pp. 117-125.

[30] D.D. Brllmann, B. dHoedt, The modulation transfer function and signalto-noise ratio of different digital filters: a technical approach, Dentomaxillofacial Radiol. (2014).

[31] F. Lettner, C. Grossauer, C. Holzmann, Mobile interaction analysis: towards a novel concept for interaction sequence mining, in: Proc. 16th Int. Conf. Human-Computer Interact. with Mob. Devices Serv., ACM, 2014: pp. 359-368.

[32] Y.-C. Chen, K.-M. Chao, On the generalized constrained longest common subsequence problems, J. Comb. Optim. 21 (2011) 383-392.

[33] U. Von Luxburg, A tutorial on spectral clustering, Stat. Comput. 17 (2007) 395-416.

[34] R.A. Horn, C.R. Johnson, Topics in matrix analysis, Cambridge University Press, 1994.

[35] Z. Hu, G. Pan, Y. Wang, Z. Wu, Spectral Sparse Representation for Clustering: Evolved from PCA, K-means, Laplacian Eigenmap, and Ratio Cut, arXiv Prepr. arXiv1403.6290. (2014).

[36] N. Xue, H. He, J. Liu, Q. Zheng, T. Ma, J. Ruan, B. Dong, Probabilistic Modeling Towards Understanding the Power Law Distribution of Video Viewing Behavior in Large-Scale e-Learning, in: Trust. 2015 IEEE, IEEE, 2015: pp. 136-142. 